\title{
SOME CONSIDERATIONS ON THE DERIVATION OF THE NONLINEAR QUANTUM BOLTZMANN EQUATION.
}

\author{
D. Benedetto*, F. Castella ${ }^{+}$, R. Esposito\# and M. Pulvirenti*
}

\begin{abstract}
In this paper we analyze a system of $N$ identical quantum particles in a weakcoupling regime. The time evolution of the Wigner transform of the one-particle reduced density matrix is represented by means of a perturbative series. The expansion is obtained upon iterating the Duhamel formula. For short times, we rigorously prove that a subseries of the latter, converges to the solution of the Boltzmann equation which is physically relevant in the context. In particular, we recover the transition rate as it is predicted by Fermi's Golden Rule. However, we are not able to prove that the quantity neglected while retaining a subseries of the complete original perturbative expansion, indeed vanishes in the limit: we only give plausibility arguments in this direction. The present study holds in any space dimension $d \geq 2$.
\end{abstract}

\section{INTRODUCTION.}

A large quantum particle system in a rarefaction regime should be described by a Boltzmann equation. However, while the rigorous validity of the Boltzmann equation has been proved for classical systems for short times [L] or globally in time for special situations [IP] (see Ref. [CIP] for further comments), there is no rigorous analysis for the equivalent quantum systems.

The problem is physically relevant because quantum effects, although usually negligible at ordinary temperatures (except for few light molecules), happen to play a rôle in the applications at mesoscopic level. We refer, for example, to the treatment of electron gases in semiconductors (for physical references, see the textbooks $[\mathrm{RV}],[\mathrm{AM}],[\mathrm{Ch}]$, as well as $[\mathrm{Bo}],[\mathrm{CTDL}]$ - see also the articles [Fi] or $[\mathrm{Co}]$ - see $[\mathrm{MRS}]$ for a mathematically oriented presentation). Therefore, establishing a well founded quantum kinetic theory is certainly interesting not only from a conceptual viewpoint but also from a practical one. In fact, kinetic descriptions for quantum systems, beside dilute gases, include dense weakly interacting systems, as e.g. the electron gas in semiconductors, whose classical analogues rather yield diffusion processes.

One pragmatic way to introduce the Quantum Boltzmann equation (see e.g. [CC]) is to solve the scattering problem in Quantum Mechanics and then to replace, in the classical Boltzmann equation, the classical cross section with the Quantum one.

A better logically founded approach is to derive an evolution equation for the Wigner transform of a quantum state associated to a dilute particle system. Working on this equation, one can hope to recover, at the quantum level, the same physical arguments

*Dipartimento di Matematica Università di Roma 'La Sapienza', P.le A. Moro, 5, 00185 Roma, Italy + IRMAR Université de Rennes 1, Campus de Beaulieu 35042 Rennes-Cedex France \#Centro Linceo Interdisciplinare "Beniamino Segre", Via della Lungara 10, 00165 Roma, and Dipartimento di Matematica pura ed applicata, Università di L'Aquila, Coppito, 67100 L'Aquila, Italy 
than those used at the classical level to obtain propagation of chaos and a suitable kinetic description for the one particle distribution function. This is the strategy we adopt in the present paper to treat quantum $N$-particle systems. We refer to the textbook [CIP], or the article $[\mathrm{L}]$ for the analysis of the classical case.

Summarizing, in the present paper, we consider a quantum $N$-particle system and represent the time evolution of the one-particle Wigner function, when $N \rightarrow \infty$ in the weak-coupling limit, in terms of a perturbative series expansion. On the basis of some heuristic arguments developed in the next section, we neglect some terms and consider only a subseries which is proven to converge, for short times, to the solution of the Boltzmann equation with a suitable cross section computed by quantum rules (namely the Fermi Golden Rule). Therefore, the present analysis is not a rigorous derivation of the Quantum Boltzmann equation, but we hope it constitutes a step in this direction. Our main result is Theorem 3.1 below. Although we work in dimension 3 the present statements are easily extended in any space dimension $d \geq 2$. Our analysis heavily relies on stationary phase computations (see Proposition 3.3), as well as appropriate representations of the various solutions of the hierarchies we need to handle. We leave further comments to the last section and conclude the present one by establishing the model and the scaling.

We consider a $N$-particle quantum system in $\mathbb{R}^{3}$. We assume the mass of the particles, as well as $\hbar$, to be one. The interaction is described by a two-body potential $\phi$ so that the potential energy is:

$$
U\left(x_{1} \ldots x_{N}\right)=\sum_{i<j} \phi\left(x_{i}-x_{j}\right) .
$$

The Schrödinger equation reads:

$$
i \partial_{t} \Psi\left(X_{N}, t\right)=-\frac{1}{2} \Delta_{N} \Psi\left(X_{N}, t\right)+U\left(X_{N}\right) \Psi\left(X_{N}, t\right)
$$

where $\Delta_{N}=\sum_{i=1}^{N} \Delta_{i}, \Delta_{i}$ is the Laplacian with respect to the $x_{i}$ variables, and $X_{N}$ is a shorthand notation for $x_{1} \ldots x_{N}$.

We rescale the equation according to the hyperbolic space-time scaling

$$
x \rightarrow \varepsilon x, \quad t \rightarrow \varepsilon t
$$

and simultaneously we rescale also the potential $\phi \rightarrow \sqrt{\varepsilon} \phi$. Hence the resulting equation is

$$
i \varepsilon \partial_{t} \Psi^{\varepsilon}\left(X_{N}, t\right)=-\frac{\varepsilon^{2}}{2} \Delta_{N} \Psi^{\varepsilon}\left(X_{N}, t\right)+U_{\varepsilon}\left(X_{N}\right) \Psi^{\varepsilon}\left(X_{N}, t\right),
$$

where

$$
U_{\varepsilon}\left(x_{1} \ldots x_{N}\right)=\sum_{i<j} \phi_{\varepsilon}\left(x_{i}-x_{j}\right)
$$

and

$$
\phi_{\varepsilon}=\sqrt{\varepsilon} \phi\left(\frac{x}{\varepsilon}\right) .
$$

Note that $\Psi^{\varepsilon}\left(X_{N}, t\right)$ is fully determined by Eq. (1.4) and the initial datum which will be specified later on. 
We want to analyze the limit $\varepsilon \rightarrow 0$ in the above equations, while keeping

$$
N=\varepsilon^{-3} .
$$

This kind of limit is usually called weak-coupling limit. Another possible scaling to be considered is the low-density limit. In this case $\phi$ is unscaled but $N=O\left(\varepsilon^{-2}\right)$. In the classical context this is nothing but the Boltzmann-Grad limit (see e.g. [CIP]). In the present paper we will only be concerned with the weak coupling limit which is, to some extent, technically easier.

We now introduce the Wigner function:

$$
W^{N}\left(X_{N}, V_{N}\right)=\left(\frac{1}{2 \pi}\right)^{3 N} \int d Y_{N} e^{i Y_{N} \cdot V_{N}} \bar{\Psi}^{\varepsilon}\left(X_{N}+\frac{\varepsilon}{2} Y_{N}\right) \Psi^{\varepsilon}\left(X_{N}-\frac{\varepsilon}{2} Y_{N}\right) .
$$

A standard computation yields:

$$
\left(\partial_{t}+V_{N} \cdot \nabla_{N}\right) W^{N}\left(X_{N}, V_{N}\right)=\frac{1}{\sqrt{\varepsilon}}\left(T_{N}^{\varepsilon} W^{N}\right)\left(X_{N}, V_{N}\right)
$$

where $V_{N} \cdot \nabla_{N}=\sum_{i=1}^{N} v_{i} \cdot \nabla_{x_{i}}$ and $\left(\partial_{t}+V_{N} \cdot \nabla_{N}\right)$ is the usual free stream operator. Also, we have introduced

$$
\left(T_{N}^{\varepsilon} W^{N}\right)\left(X_{N}, V_{N}\right)=\sum_{0<k<\ell \leq N}\left(T_{k, \ell}^{\varepsilon} W^{N}\right)\left(X_{N}, V_{N}\right)
$$

with

$$
\begin{gathered}
\left(T_{k, \ell}^{\varepsilon} W^{N}\right)\left(X_{N}, V_{N}\right)=\frac{1}{i}\left(\frac{1}{2 \pi}\right)^{3 N} \int d Y_{N} \int d V_{N}^{\prime} \mathrm{e}^{i Y_{N} \cdot\left(V_{N}-V_{N}^{\prime}\right)} W^{N}\left(X_{N}, V_{N}^{\prime}\right) \\
{\left[\phi\left(\frac{x_{k}-x_{\ell}}{\varepsilon}-\frac{1}{2}\left(y_{k}-y_{\ell}\right)\right)-\phi\left(\frac{x_{k}-x_{\ell}}{\varepsilon}+\frac{1}{2}\left(y_{k}-y_{\ell}\right)\right)\right] .}
\end{gathered}
$$

In other words,

$$
\begin{aligned}
& \left(T_{k, \ell}^{\varepsilon} W^{N}\right)\left(X_{N}, V_{N}\right)=-i \sum_{\sigma= \pm 1} \sigma \int \frac{d h}{(2 \pi)^{3}} \hat{\phi}(h) \mathrm{e}^{i \frac{h}{\varepsilon}\left(x_{k}-x_{\ell}\right)} \\
& W^{N}\left(x_{1}, v_{1}, \ldots, x_{k}, v_{k}-\frac{\sigma h}{2}, \ldots, x_{\ell}, v_{\ell}+\frac{\sigma h}{2}, \ldots, x_{N}, v_{N}\right) .
\end{aligned}
$$

The operator $T_{k, \ell}^{\varepsilon}$ describes the "collision" of particle $k$ with particle $\ell$, and the total operator $T_{N}^{\varepsilon}$ takes all possible "collisions" into account. Here and below, $\hat{f}$ denotes the Fourier transform of $f$, normalized as follows:

$$
\begin{gathered}
\hat{f}(h)=\left(\mathcal{F}_{x} f\right)(h)=\int_{\mathbb{R}^{3}} d x f(x) \mathrm{e}^{-i h \cdot x}, \\
f(x)=\int_{\mathbb{R}^{3}} \frac{d h}{(2 \pi)^{3}} \hat{f}(h) \mathrm{e}^{i h \cdot x} .
\end{gathered}
$$


We now introduce the Wigner transform of the partial traces according to the formula, for $j=1 \ldots, N-1$ :

$$
f_{j}^{N}\left(X_{j}, V_{j}\right)=\int d x_{j+1} \ldots d x_{N} \int d v_{j+1} \ldots d v_{N} W^{N}\left(X_{j}, x_{j+1} \ldots x_{N} ; V_{j}, v_{j+1} \ldots v_{N}\right)
$$

Obviously, we set $f_{N}^{N}=W^{N}$.

From now on we shall suppose that, due to the fact that the particles are identical, the objects which we have introduced $\left(\Psi^{\varepsilon}, W^{N}, f_{j}^{N}\right)$ are all symmetric in the exchange of particles.

Proceeding as in the derivation of the BBKGY hierarchy for classical systems (see [CIP]), we readily arrive at the following hierarchy of equations (for $1 \leq j \leq N$ ):

$$
\left(\partial_{t}+\sum_{k=1}^{j} v_{k} \cdot \nabla_{k}\right) f_{j}^{N}=\frac{1}{\sqrt{\varepsilon}} T_{j}^{\varepsilon} f_{j}+\frac{N-j}{\sqrt{\varepsilon}} C_{j+1}^{\varepsilon} f_{j+1}^{N} .
$$

The operator $C_{j+1}^{\varepsilon}$ is defined as:

$$
C_{j+1}^{\varepsilon}=\sum_{k=1}^{j} C_{k, j+1}^{\varepsilon}
$$

and

$$
\begin{gathered}
C_{k, j+1}^{\varepsilon} f_{j+1}\left(x_{1} \ldots x_{j} ; v_{1} \ldots v_{j}\right)=-i \sum_{\sigma= \pm 1} \sigma \int \frac{d h}{(2 \pi)^{3}} \int d x_{j+1} \int d v_{j+1} \hat{\phi}(h) \\
e^{i \frac{h}{\varepsilon}\left(x_{k}-x_{j+1}\right)} f_{j+1}\left(x_{1}, x_{2}, \ldots, x_{j+1}, v_{1}, \ldots, v_{k}-\sigma \frac{h}{2}, \ldots, v_{j+1}+\sigma \frac{h}{2}\right) .
\end{gathered}
$$

The operator $C_{k, j+1}^{\varepsilon}$ describes the "collision" of particle $k$, belonging to the $j$-particle subsystem, with a generic particle outside the subsystem, conventionally denoted by the number $j+1$ (this numbering uses the fact that all the particles are identical). The total operator $C_{j+1}^{\varepsilon}$ takes into account all such collisions. As usual ([CIP]), equation (1.15) shows that the dynamics of the $j$-particle subsystem is governed by three effects: the freestream operator, the collisions "inside" the subsystem (the $T$ term), and the collisions with particles "outside" the subsystem (the $C$ term).

We fix the initial value $\left\{f_{j}^{0}\right\}_{j=1}^{N}$ of the solution $\left\{f_{j}^{N}(t)\right\}_{j 1}^{N}$ and we assume for simplicity that $\left\{f_{j}^{0}\right\}_{j=1}^{N}$ is factorized, that is, for all $j=1, N$

$$
f_{j}^{0}=f_{0}^{\otimes j}
$$

where $f_{0}$ is a one-particle Wigner function which we assume also to be a probability distribution. We remind that the quantum state, whose Wigner transform is a general positive $f_{0}$, is not in general a wave function but rather a density matrix. As a consequence the evolution equation we have to use is not the Schrödinger equation (1.2) but rather the Heisenberg equation for the density matrix. In both cases the corresponding Wigner equation is $(1.9)$. 
One can try to handle the hierarchy (1.15) as for the Boltzmann-Grad limit for classical systems, namely to study the asymptotic behavior of the solution expressed in terms of the series expansion for $1 \leq j \leq N$, obtained upon iterating the Duhamel formula,

$$
\begin{gathered}
f_{j}^{N}(t)=\sum_{n=0}^{N-j} \frac{(N-j) \ldots(N-j-n)}{(\sqrt{\varepsilon})^{n}} \int_{0}^{t} d t_{1} \cdots \int_{0}^{t_{n-1}} d t_{n} S_{i n t}^{\varepsilon}\left(t-t_{1}\right) C_{j+1}^{\varepsilon} \\
S_{i n t}^{\varepsilon}\left(t_{1}-t_{2}\right) C_{j+2}^{\varepsilon} \ldots S_{i n t}^{\varepsilon}\left(t_{n-1}-t_{n}\right) C_{j+n}^{\varepsilon} S_{i n t}^{\varepsilon}\left(t_{n}\right) f_{j+n}^{0} .
\end{gathered}
$$

Here $S_{i n t}^{\varepsilon}(t) f_{j}$ is the $j$-particle interacting flow, namely the solution to the initial value problem:

$$
\left\{\begin{array}{l}
\left(\partial_{t}+V_{j} \cdot \nabla_{j}\right) S_{i n t}^{\varepsilon}(t) f_{j}=\frac{1}{\sqrt{\varepsilon}} T_{j}^{\varepsilon} S_{i n t}^{\varepsilon}(t) f_{j}, \\
S_{i n t}^{\varepsilon}(0) f_{j}=f_{j} .
\end{array}\right.
$$

If we expand $S_{i n t}^{\varepsilon}(t)$ as a perturbation of the free flow $S(t)$ defined as

$$
\left(S(t) f_{j}\right)\left(X_{j}, V_{j}\right)=f_{j}\left(X_{j}-V_{j} t, V_{j}\right)
$$

we find

$$
\begin{gathered}
S_{i n t}^{\varepsilon}(t) f_{j}=S(t) f_{j}+\sum_{m \geq 0} \frac{1}{(\sqrt{\varepsilon})^{m}} \int_{0}^{t} d \tau_{1} \int_{0}^{\tau_{1}} d \tau_{2} \cdots \int_{0}^{\tau_{m-1}} d \tau_{m} S\left(t-\tau_{1}\right) T_{j}^{\varepsilon} \\
S\left(\tau_{1}-\tau_{2}\right) T_{j}^{\varepsilon} \ldots S\left(\tau_{m-1}-\tau_{m}\right) T_{j}^{\varepsilon} S\left(\tau_{m}\right) f_{j} .
\end{gathered}
$$

Inserting (1.22) into (1.19), the resulting series contains a huge number of terms. However, we claim that many of these contributions are negligible in the limit. In the next section, we shall give heuristic arguments in that direction. We first make our point more precise. We write shortly (1.19) and (1.22) as

$$
\begin{aligned}
f_{j}^{N} & =\sum_{n=0}^{N-j} S_{i n t}^{\varepsilon} \widetilde{C}_{j+1}^{\varepsilon} S_{i n t}^{\varepsilon} \widetilde{C}_{j+2}^{\varepsilon} \cdots S_{i n t}^{\varepsilon} \widetilde{C}_{j+n}^{\varepsilon} S_{i n t}^{\varepsilon}, \\
S_{i n t}^{\varepsilon} & =\sum_{m \geq 0}\left(S \widetilde{T}_{j}\right)^{m} S,
\end{aligned}
$$

with an obvious abuse of notation. Here the tilde above $C$ and $T$ terms absorbs the normalization by $N / \sqrt{\varepsilon}$ in (1.19), respectively $1 / \sqrt{\varepsilon}$ in (1.22). Now, the insertion of (1.19) into (1.22) readily gives

$$
\begin{gathered}
f_{j}^{N}=\sum_{n=0}^{N-j} \sum_{m_{0} \geq 0} \sum_{m_{1} \geq 0} \cdots \sum_{m_{n} \geq 0}\left(S \widetilde{T}_{j}\right)^{m_{0}} S \widetilde{C}_{j+1}^{\varepsilon}\left(S \widetilde{T}_{j+1}\right)^{m_{1}} S \widetilde{C}_{j+2}^{\varepsilon} \cdots \\
\cdots\left(S \widetilde{T}_{j+n-1}\right)^{m_{n-1}} S \widetilde{C}_{j+n}^{\varepsilon}\left(S \widetilde{T}_{j+n}\right)^{m_{n}} S
\end{gathered}
$$

In view of the expansion (1.23) of $f_{j}^{N}$, we first claim that all the relevant terms in (1.23) is that corresponding to

$$
m_{0}=0, m_{1}=m_{2}=\cdots=m_{n}=1 .
$$


Hence as $\varepsilon \rightarrow 0, f_{j}^{N}$ is asymptotic to,

$$
f_{j}^{N} \sim \sum_{n=0}^{N-j} S \widetilde{C}_{j+1}^{\varepsilon} S \widetilde{T}_{j+1} S \widetilde{C}_{j+2}^{\varepsilon} S \widetilde{T}_{j+2} \cdots S \widetilde{T}_{j+n-1} S \widetilde{C}_{j+n}^{\varepsilon} S \widetilde{T}_{j+n} S
$$

On the more, expanding each "collision" term in (1.24) into $C_{j+1}^{\varepsilon}=\sum_{r=1}^{j} C_{r, j+1}^{\varepsilon}$, and $T_{j}^{\varepsilon}=$ $\sum_{r, \ell=1}^{j} T_{r, \ell}^{\varepsilon}$, we also claim that $f_{j}^{N}$ is in fact asymptotic to,

$$
f_{j}^{N} \sim \sum_{n=0}^{N-j} \sum_{r_{1}=1}^{j} S \widetilde{C}_{r_{1}, j+1}^{\varepsilon} S \widetilde{T}_{r_{1}, j+1} \sum_{r_{2}=1}^{j+1} S \widetilde{C}_{r_{2}, j+2}^{\varepsilon} S \widetilde{T}_{r_{2}, j+2} \cdots \sum_{r_{n}=1}^{j+n-1} S \widetilde{C}_{r_{n}, j+n}^{\varepsilon} S \widetilde{T}_{r_{n}, j+n} S .
$$

In other terms, we claim that the dynamics of the $j$-particle subsystem is only made up of collision/recollision events in the asymptotics $\varepsilon \rightarrow 0$.

Summarizing the above claims, we define the sequence $\left\{\tilde{f}_{j}^{N}\right\}_{j=1}^{N}$ by

$$
\begin{gathered}
\tilde{f}_{j}^{N}(t)=S(t) f_{j}^{0}+\sum_{n=1}^{N-j} \frac{(N-j) \ldots(N-j-n)}{(\sqrt{\varepsilon})^{n}} \int_{0}^{t} d t_{1} \int_{0}^{t_{1}} d \tau_{1} \int_{0}^{\tau_{1}} d t_{2} \int_{0}^{t_{2}} d \tau_{2} \ldots \\
\cdots \int_{0}^{t_{n-1}} d t_{n} \int_{0}^{t_{n}} d \tau_{n} \sum_{r_{1}=1}^{j} \sum_{r_{2}=1}^{j+1} \cdots \sum_{r_{n}=1}^{j+n-1} S\left(t-t_{1}\right) C_{r_{1}, j+1}^{\varepsilon} S\left(t_{1}-\tau_{1}\right) T_{r_{1}, j+1}^{\varepsilon} \\
S\left(\tau_{1}-t_{2}\right) C_{r_{2}, j+2}^{\varepsilon} S\left(t_{2}-\tau_{2}\right) T_{r_{1}, j+1}^{\varepsilon} \cdots \\
\cdots S\left(\tau_{n-1}-t_{n}\right) C_{r_{n}, j+n}^{\varepsilon} S\left(t_{n}-\tau_{n}\right) T_{r_{n}, j+n}^{\varepsilon} S\left(\tau_{n}\right) f_{j+n}^{0} .
\end{gathered}
$$

Obviously $\tilde{f}_{j}^{N}$ is a subseries of the series expansion (1.23) defining the true value $f_{j}^{N}$. It relates the value of the right-hand-side of (1.25). The remainder part of this paper is dedicated to the rigorous proof that $\tilde{f}_{j}^{\varepsilon}(t)$ converge to $(f(t))^{\otimes j}$ for short times, where $f(t)$ solves the Boltzmann equation with the suitable cross-section. The result will be precisely established and proved in Section 3.

Note that unfortunately, we are not able to rigorously prove the asymptotics (1.25): neither can we prove reasonable uniform bounds on the relevant series expansions, nor can we even prove that the asymptotics (1.23) holds term-by-term.

\section{Heuristic CONSIDERATions.}

In this section, we give some reasons justifying the claimed asymptotics (1.24) and (1.25). These are illustrated upon analyzing the lower order terms in the true expansion (1.23). This section thus heuristically justifies the fact that we restrict ourselves with the mere analysis of $\tilde{f}_{j}^{N}$ in the present paper.

Also, we analyze the term corresponding to $n=1$ in the expansion (1.25) defining $\tilde{f}_{j}^{N}$. We prepare in this way the analysis of the complete series expansion performed in the next section. 
To have an idea of the various orders of magnitude of the terms appearing in (1.19) once $S_{\text {int }}^{\varepsilon}$ is expanded (see (1.23)), we consider the first two terms in (1.19) expanding $S_{\text {int }}^{\varepsilon}$ up to the first order. As a result we have the following five terms which we are going to analyze:

$$
\begin{gathered}
\mathcal{I}_{0}=S(t) f_{j}^{0}, \\
\mathcal{I}_{1}=\frac{N-j}{\sqrt{\varepsilon}} \int_{0}^{t} d t_{1} S\left(t-t_{1}\right) C_{j+1}^{\varepsilon} S\left(t_{1}\right) f_{j+1}^{0}, \\
\mathcal{I}_{2}=\frac{1}{\sqrt{\varepsilon}} \int_{0}^{t} d \tau_{1} S\left(t-\tau_{1}\right) T_{j}^{\varepsilon} S\left(\tau_{1}\right) f_{j}^{0}, \\
\mathcal{I}_{3}=\frac{N-j}{\varepsilon} \int_{0}^{t} d \tau_{1} \int_{0}^{\tau_{1}} d t_{1} S\left(t-\tau_{1}\right) T_{j}^{\varepsilon} S\left(\tau_{1}-t_{1}\right) C_{j+1}^{\varepsilon} S\left(t_{1}\right) f_{j+1}^{0}, \\
\mathcal{I}_{4}=\frac{N-j}{\sqrt{\varepsilon}} \int_{0}^{t} d t_{1} \int_{0}^{t_{1}} d \tau_{1} S\left(t-t_{1}\right) C_{j+1}^{\varepsilon} S\left(t_{1}-\tau_{1}\right) T_{j+1}^{\varepsilon} S\left(\tau_{1}\right) f_{j+1}^{0} \\
=\sum_{r=1}^{j} \sum_{1 \leq s<\ell \leq j+1} \mathcal{I}_{4}^{r, \ell, s},
\end{gathered}
$$

where

$$
\mathcal{I}_{4}^{r, \ell, s}=\frac{N-j}{\sqrt{\varepsilon}} \int_{0}^{t} d t_{1} \int_{0}^{t_{1}} d \tau_{1} S\left(t-t_{1}\right) C_{r, j+1}^{\varepsilon} S\left(t_{1}-\tau_{1}\right) T_{\ell, s}^{\varepsilon} S\left(\tau_{1}\right) f_{j+1}^{0} .
$$

As we shall see, the terms $\mathcal{I}_{i}, i=1,2,3$ are negligible in the limit $\varepsilon \rightarrow 0$. This illustrates (on a particular example) the claim (1.24) above. Also, all the contributions to $\mathcal{I}_{4}$ but that for $r=\ell$ and $s=j+1$, corresponding to a collision/recollision event, are equally vanishing. This illustrates the claim (1.25) above.

Clearly $\mathcal{I}_{0}$ does not require any asymptotic analysis.

For $\mathcal{I}_{1}$ we have:

$$
\begin{aligned}
& \mathcal{I}_{1}=\frac{N-j}{\sqrt{\varepsilon}} \sum_{r=1}^{j} \sum_{\sigma= \pm 1} \sigma \int_{0}^{t} d t_{1} \int d x_{j+1} \int d v_{j+1} \int \frac{d h}{(2 \pi)^{3}} \hat{\phi}(h) \mathrm{e}^{i \frac{h}{\varepsilon} \cdot\left(x_{r}-v_{r}\left(t-t_{1}\right)-x_{j+1}\right)} \\
& f_{j+1}^{0}\left(x_{1}-v_{1} t, \ldots, x_{r}-v_{r} t+\frac{\sigma h}{2} t_{1}, \ldots, x_{j+1}-v_{j+1} t_{1}-\frac{\sigma h}{2} t_{1},\right. \\
& \left.\quad v_{1}, \ldots, v_{r}-\frac{\sigma h}{2}, \ldots, v_{j+1}\right) .
\end{aligned}
$$

Now, upon using the stationary phase Theorem for the variables $x_{j+1}$ and $h$ in formula (2.7), we readily obtain the following asymptotics, valid if $f_{j+1}^{0}$ and $\phi$ are smooth and decaying enough (which we assume),

$$
\begin{gathered}
\mathcal{I}_{1}=\frac{N-j}{\sqrt{\varepsilon}} \times\left(i^{3} \varepsilon^{3} \times \sum_{r=1}^{j} \sum_{\sigma= \pm 1} \sigma \int_{0}^{t} d t_{1} \int d v_{j+1} \hat{\phi}(0)\right. \\
f_{j+1}^{0}\left(x_{1}-v_{1} t, \ldots, x_{r}-v_{r} t, \ldots, x_{r}-v_{r}\left(t-t_{1}\right)-v_{j+1} t_{1},\right. \\
\left.\left.v_{1}, \ldots, v_{r}, \ldots, v_{j+1}\right)+O\left(\varepsilon^{4}\right)\right) .
\end{gathered}
$$


In other words, the integral in (2.7) concentrates on the set $x_{j+1}=x_{r}-v_{r}\left(t-t_{1}\right), h=0$, as $\varepsilon \rightarrow 0$, and it has size $\varepsilon^{3}+O\left(\varepsilon^{4}\right)$. Recall indeed that, $\psi(x)$ being a smooth function of $x \in \mathbb{R}^{d}$, and $\varphi(x)$ being a smooth phase such that $\nabla_{x} \varphi(x)=0$ iff $x=0$, and such that the Hessian at $x=0, D_{x, x}^{2} \varphi(0)$, is invertible, then the stationary phase theorem states the asymptotics (see $[\mathrm{Hö}]$ )

$$
\int_{\mathbb{R}^{d}} d x \exp \left(\frac{i}{\varepsilon} \varphi(x)\right) \psi(x)=\varepsilon^{\frac{d}{2}} \frac{(2 i \pi)^{d / 2}}{\left(\operatorname{det} D_{x, x}^{2} \varphi(0)\right)^{1 / 2}} \psi(0)+O\left(\varepsilon^{1+\frac{d}{2}}\right) .
$$

In the case $(2.7)$, we have $d=6$. At this stage, we observe that the main contribution in (2.8) vanishes, due to the sum over $\sigma= \pm 1$. Hence we recover,

$$
\mathcal{I}_{1}=O\left(N \varepsilon^{3} \frac{\varepsilon}{\varepsilon^{1 / 2}}\right)=O\left(\varepsilon^{1 / 2}\right)
$$

For $\mathcal{I}_{2}$ we have:

$$
\begin{gathered}
\mathcal{I}_{2}=\frac{1}{\sqrt{\varepsilon}} \sum_{1 \leq r<s \leq j} \sum_{\sigma= \pm 1} \sigma \int_{0}^{t} d \tau_{1} \int \frac{d h}{(2 \pi)^{3}} \hat{\phi}(h) \mathrm{e}^{i \frac{h}{\varepsilon} \cdot\left(\left(x_{r}-x_{s}\right)-\left(v_{r}-v_{s}\right)\left(t-\tau_{1}\right)\right)} \\
f_{j}^{0}\left(x_{1}-v_{1} t, \ldots, x_{r}-v_{r} t+\frac{\sigma h}{2} \tau_{1}, \ldots, x_{s}-v_{s} t-\frac{\sigma h}{2} \tau_{1}, \ldots, x_{j}-v_{j} t\right. \\
\left.v_{1}, \ldots, v_{r}-\frac{\sigma h}{2}, \ldots, v_{s}+\frac{\sigma h}{2}, \ldots, v_{j}\right) .
\end{gathered}
$$

Now, we compute the limiting behavior of $\mathcal{I}_{2}$ in a weak sense. Hence we test formula (2.9) against a smooth function $\psi\left(x_{1}, \cdots, x_{j}, v_{1}, \cdots, v_{j}\right)$. We obtain, using the stationary phase theorem in the variables $x_{r}$ and $h$ (one could use the variables $x_{s}$ and $h$ as well)

$$
\int d x_{1} \ldots d x_{j} d v_{1} \ldots d v_{j} \mathcal{I}_{2} \psi\left(x_{1}, \ldots, x_{j}, v_{1}, \ldots, v_{j}\right)=O\left(\frac{\varepsilon^{3}}{\varepsilon^{1 / 2}}\right)=O\left(\varepsilon^{5 / 2}\right) .
$$

This again assumes some smoothness and decay assumptions on $f_{j}^{0}$.

The term $\mathcal{I}_{3}$ can be treated in the same way. We write,

$$
\mathcal{I}_{3}=\frac{N-j}{\varepsilon} \sum_{1 \leq r<s \leq j} \sum_{\ell=1}^{j} \int_{0}^{t} d \tau_{1} \int_{0}^{\tau_{1}} d t_{1} S\left(t-\tau_{1}\right) T_{r, s}^{\varepsilon} S\left(\tau_{1}-t_{1}\right) C_{\ell, j+1}^{\varepsilon} S\left(t_{1}\right) f_{j+1}^{0} .
$$

Here, as in formulae (2.7) and (2.9) defining $\mathcal{I}_{1}$ and $\mathcal{I}_{2}$, the $T$ term in (2.10) induces an integration over a variable which we call $k_{1} \in \mathbb{R}^{3}$ (playing the rôle of $h$ in formula (1.11)), and the $C$ term induces an integration over variables which we call $h_{1} \in \mathbb{R}^{3}$ (playing the rôle of $h$ in formula (1.17)), as well as $x_{j+1} \in \mathbb{R}^{3}$ and $v_{j+1} \in \mathbb{R}^{3}$. Now, for each value of $r$, $s$, and $\ell$, the corresponding term in formula (2.10) is tested against a smooth function $\psi$, as we did for $\mathcal{I}_{2}$. The result involves an integral over all variables $x_{1}, \ldots, x_{j+1}, v_{1}, \ldots$, $v_{j+1}, h_{1}$ and $k_{1}$. As in (2.9), we now apply the stationary phase theorem. More precisely, if $r \neq \ell$, we use the stationary phase in the variables $k_{1}$ and $x_{r}$ (to handle the $T$ term), as well as $h_{1}$ and $x_{j+1}$ (to handle the $C$ term). In the case $r=\ell$, we rather use the variables 
$k_{1}$ and $x_{s}$ for the $T$ term, as well as $h_{1}$ and $x_{j+1}$ for the $C$ term. We refer to the treatment of $\mathcal{I}_{4}$ below for details. Using this approach, we eventually obtain,

$$
\mathcal{I}_{3}=O\left(\frac{N-j}{\varepsilon} \varepsilon^{6}\right)=O\left(\varepsilon^{2}\right)
$$

in a weak sense (i.e. in the sense of distributions in $x_{1}, \ldots, x_{j}, v_{1}, \ldots, v_{j}$ ). This again requires some smoothness and decay properties for the function $f_{j+1}^{0}$ as well as $\phi$.

To treat the last term $\mathcal{I}_{4}$ we have to distinguish various cases:

1) $\{r, j+1\} \cap\{\ell, s\}=\emptyset$,

2) $r=\ell$ and $s \neq j+1$,

3) $r \neq \ell, s=j+1$,

4) $r=\ell, s=j+1$,

and treat them separately. Item (4) obviously corresponds to a collision/recollision event, and this is the dominant term that we want to keep in the limit, while replacing the true expansion (1.23) by the reduced series (1.25).

As regards case 1), we write,

$$
\begin{gathered}
\mathcal{I}_{4}^{r, \ell, s}=\frac{N-j}{\varepsilon} \sum_{\sigma_{1}, \sigma_{1}^{\prime}= \pm 1} \sigma_{1} \sigma_{1}^{\prime} \int_{0}^{t} d t_{1} \int_{0}^{t_{1}} d \tau_{1} \int d x_{j+1} d v_{j+1} \int \frac{d h_{1}}{(2 \pi)^{3}} \int \frac{d k_{1}}{(2 \pi)^{3}} \\
\hat{\phi}\left(h_{1}\right) \hat{\phi}\left(k_{1}\right) \mathrm{e}^{i \frac{h_{1}}{\varepsilon} \cdot\left(x_{r}-x_{j+1}-v_{r}\left(t-t_{1}\right)\right)} \mathrm{e}^{i \frac{k_{1}}{\varepsilon} \cdot\left(x_{\ell}-x_{s}-\left(v_{\ell}-v_{s}\right)\left(t-\tau_{1}\right)\right)} f_{j+1}^{0}\left(x_{1}-v_{1} t,\right. \\
x_{\ell}-v_{\ell} t+\frac{\sigma_{1}^{\prime} k_{1}}{2} \tau_{1}, \ldots, x_{s}-v_{s} t-\frac{\sigma_{1}^{\prime} k_{1}}{2} \tau_{1}, \ldots, x_{r}-v_{r} t+\frac{\sigma_{1} h_{1}}{2} t_{1}, \ldots, \\
x_{j+1}-v_{j+1} t-\frac{\sigma_{1} h_{1}}{2} t_{1} ; v_{1}, \ldots, v_{\ell}-\frac{\sigma_{1}^{\prime} k_{1}}{2}, \ldots, v_{s}+\frac{\sigma_{1}^{\prime} k_{1}}{2}, \ldots, v_{r}-\frac{\sigma_{1} h_{1}}{2}, \\
\left.\ldots, v_{j+1}+\frac{\sigma_{1} h_{1}}{2}\right) .
\end{gathered}
$$

As before, we test formula (2.11) against a smooth function $\psi$, and the stationary phase theorem in the variables $h_{1}, x_{j+1}$ as well as $k_{1}, x_{\ell}$, readily gives in this case,

$$
\mathcal{I}_{4}^{r, \ell, s}=O\left(\frac{N-j}{\varepsilon} \varepsilon^{6}\right)=O\left(\varepsilon^{2}\right)
$$

in the sense of distributions in $x_{1}, \ldots, x_{j}, v_{1}, \ldots, v_{j}$.

Case 2) follows along the same lines, except that we now use the stationary phase Theorem in the variables $h_{1}, x_{j+1}$, as well as $k_{1}, x_{s}$, in the formula,

$$
\begin{aligned}
& \mathcal{I}_{4}^{\ell, \ell, s}=\frac{N-j}{\varepsilon} \sum_{\sigma_{1}, \sigma_{1}^{\prime}= \pm 1} \sigma_{1} \sigma_{1}^{\prime} \int_{0}^{t} d t_{1} \int_{0}^{t_{1}} d \tau_{1} \int d x_{j+1} d v_{j+1} \int \frac{d h_{1}}{(2 \pi)^{3}} \int \frac{d k_{1}}{(2 \pi)^{3}} \\
& \hat{\phi}\left(h_{1}\right) \hat{\phi}\left(k_{1}\right) \mathrm{e}^{i \frac{h_{1}}{\varepsilon} \cdot\left(x_{\ell}-x_{j+1}-v_{\ell}\left(t-t_{1}\right)\right)} \mathrm{e}^{i \frac{k_{1}}{\varepsilon} \cdot\left(x_{\ell}-x_{s}-\left(v_{\ell}-v_{s}\right)\left(t-t_{1}\right)-\left(v_{\ell}-\frac{\sigma_{1} h_{1}}{2}-v_{s}\right)\left(t_{1}-\tau_{1}\right)\right)} \\
& f_{j+1}^{0}\left(x_{1}-v_{1} t, x_{\ell}-v_{\ell} t+\frac{\sigma_{1} h_{1}}{2} t_{1}+\frac{\sigma_{1}^{\prime} k_{1}}{2} \tau_{1}, \ldots, x_{s}-v_{s} t-\frac{\sigma_{1}^{\prime} k_{1}}{2} \tau_{1}, \ldots,\right. \\
& \left.x_{j+1}-v_{j+1} t-\frac{\sigma_{1} h_{1}}{2} t_{1} ; v_{1}, \ldots, v_{\ell}-\frac{\sigma_{1} h_{1}}{2}-\frac{\sigma_{1}^{\prime} k_{1}}{2}, \ldots, v_{s}+\frac{\sigma_{1}^{\prime} k_{1}}{2}, \ldots, v_{j+1}+\frac{\sigma_{1} h_{1}}{2}\right) .
\end{aligned}
$$


Case 3) is the same, and we simply need to use stationary phase in the variables $h_{1}$, $x_{r}$, as well as $k_{1}, x_{\ell}$, in a formula analogous to (2.12). All these computation require, as usual, some smoothness and decay assumptions on $f_{j+1}^{0}$ and $\phi$.

Now, case 4) cannot be treated by a direct stationary phase approach, since the space variables (the difference $x_{\ell}-x_{j+1}$ ) in the two oscillating factors appearing in this case,

$$
\begin{aligned}
& \exp \left(i \frac{h_{1}}{\varepsilon} \cdot\left(x_{\ell}-x_{j+1}-v_{\ell}\left(t-t_{1}\right)\right)\right) \\
& \exp \left(i \frac{k_{1}}{\varepsilon} \cdot\left(x_{\ell}-x_{j+1}-\left(v_{\ell}-v_{j+1}\right)\left(t-t_{1}\right)-\left(v_{\ell}-v_{j+1}-\sigma_{1} h_{1}\right)\left(t_{1}-\tau_{1}\right)\right)\right)
\end{aligned}
$$

are the same: one cannot decouple the problem into simply applying twice the stationary phase theorem (one time for each oscillating factor) as we did before. It turns out that the present contribution is, beside $\mathcal{I}_{0}$, the only contribution $O(1)$ which survive in the limit.

We thus end up this section by carefully analyzing the limit $\varepsilon \rightarrow 0$ in case (4). The analysis performed here allows to understand in a particular case all the arguments needed for the general proof given in the next section. We write,

$$
\begin{aligned}
& \mathcal{I}_{4}^{\ell, \ell, j+1}=\frac{N-j}{\varepsilon} \sum_{\sigma_{1}, \sigma_{1}^{\prime}= \pm 1} \sigma_{1} \sigma_{1}^{\prime} \int_{0}^{t} d t_{1} \int_{0}^{t_{1}} d \tau_{1} \int d x_{j+1} d v_{j+1} \int \frac{d h_{1}}{(2 \pi)^{3}} \int \frac{d k_{1}}{(2 \pi)^{3}} \\
& \hat{\phi}\left(h_{1}\right) \hat{\phi}\left(k_{1}\right) \mathrm{e}^{i \frac{\xi_{1}}{\varepsilon} \cdot\left(x_{\ell}-x_{j+1}-v_{\ell}\left(t-t_{1}\right)\right)} \mathrm{e}^{i \frac{k_{1}}{\varepsilon} \cdot\left(x_{\ell}-x_{j+1}-\left(v_{\ell}-v_{j+1}\right)\left(t-t_{1}\right)-\left(v_{\ell}-v_{j+1}-\sigma_{1} h_{1}\right)\left(t_{1}-\tau_{1}\right)\right)} \\
& f_{j+1}^{0}\left(x_{1}-v_{1} t, \ldots, x_{\ell}-v_{\ell} t+\frac{\sigma_{1} h_{1}}{2} t_{1}+\frac{\sigma_{1}^{\prime} k_{1}}{2} \tau_{1}, \ldots, x_{j+1}-v_{j+1} t-\frac{\sigma_{1} h_{1}}{2} t_{1}-\frac{\sigma_{1}^{\prime} k_{1}}{2} \tau_{1} ;\right. \\
& \left.v_{1}, \ldots, v_{\ell}-\frac{\sigma_{1} h_{1}}{2}-\frac{\sigma_{1}^{\prime} k_{1}}{2}, \ldots, \ldots, v_{j+1}+\frac{\sigma_{1} h_{1}}{2}+\frac{\sigma_{1}^{\prime} k_{1}}{2}\right) .
\end{aligned}
$$

As explained above, one cannot make use of the oscillations of the product (2.13) by simply using the stationary phase in the variables $h_{1}, k_{1}, x_{\ell}$ and $x_{j+1}$ : one has to use the oscillations in the velocity variable $v_{j+1}$ as well. Unfortunately, the factor $\left(t_{1}-\tau_{1}\right)$ in (2.13) may vanish, thus killing the oscillation. This is the very reason for the rescaling we now perform. We make the following change of variables in (2.14)

$$
\begin{aligned}
& t_{1}-\tau_{1}=\varepsilon s_{1}, \quad\left(\text { i.e. } \tau_{1}=t_{1}-\varepsilon s_{1}\right), \\
& \xi_{1}=\left(h_{1}+k_{1}\right) / \varepsilon .
\end{aligned}
$$

The variable $s_{1}$ is the rescaled time between the first "collision" involving particles $\ell$ and $j+1$ (occurring at time $t_{1}$ ), and the recollision event (occurring at time $\tau_{1}$ ). This gives in $(2.14)$,

$$
\begin{aligned}
& \mathcal{I}_{4}^{\ell, \ell, j+1}=(N-j) \varepsilon^{3} \sum_{\sigma_{1}, \sigma_{1}^{\prime}= \pm 1} \sigma_{1} \sigma_{1}^{\prime} \int_{0}^{t} d t_{1} \int_{0}^{t_{1} / \varepsilon} d s_{1} \int d x_{j+1} d v_{j+1} \int \frac{d \xi_{1}}{(2 \pi)^{3}} \int \frac{d k_{1}}{(2 \pi)^{3}} \\
& \hat{\phi}\left(-k_{1}+\varepsilon \xi_{1}\right) \hat{\phi}\left(k_{1}\right) \mathrm{e}^{i \xi_{1} \cdot\left(x_{\ell}-x_{j+1}-v_{\ell}\left(t-t_{1}\right)\right)} \mathrm{e}^{-i s_{1} k_{1} \cdot\left(v_{\ell}-v_{j+1}-\sigma_{1} h_{1}\right)} f_{j+1}^{0}(\ldots) .
\end{aligned}
$$


Now, in order to treat the limit $\varepsilon \rightarrow 0$ in (2.16), one observes that it is of the form,

$$
A_{\varepsilon}=\int_{\mathbb{R}^{4 d}} d x d \xi d y d \eta \int_{s=0}^{1 / \varepsilon} d s \exp (i \xi \cdot x-i s \eta \cdot y) \chi(x, y, \xi, \eta)
$$

for some smooth function $\chi$. We claim that,

$$
A_{\varepsilon} \rightarrow(2 \pi)^{3} \int_{\mathbb{R}^{2 d}} d y d \eta \int_{s=0}^{+\infty} d s \exp (-i s \eta \cdot y) \chi(0,0, \xi, \eta)
$$

with the uniform bound,

$$
\begin{aligned}
\left|A_{\varepsilon}\right| \leq C\left\|\left(\mathcal{F}_{x, y} \chi\right)(\alpha, \beta, \xi, \eta)\right\|_{L^{1}\left(d \xi d \eta ; L^{\infty}(d \alpha d \beta)\right)} & \\
& +C\left\|\left(\mathcal{F}_{x, y} \chi\right)(\alpha, \beta, \xi, \eta)\right\|_{L^{1}\left(d \alpha d \beta ; L^{\infty}(d \xi d \eta)\right)} .
\end{aligned}
$$

This is merely a variant of the stationary phase Theorem, and it relies on the simple two identities,

$$
\begin{gathered}
\int d x d \xi d y d \eta \mathrm{e}^{i x \cdot \xi-i s \eta \cdot y} \chi(x, y, \xi, \eta)=\int d \xi d \eta\left(\mathcal{F}_{x, y} \chi\right)(-\xi, s \eta, \xi, \eta) \\
=s^{-d} \int d \xi d \eta\left(\mathcal{F}_{x, y} \chi\right)(-\xi, \eta, \xi, \eta / s)
\end{gathered}
$$

giving, in particular, the absolute convergence of the $d s$ integral in (2.18). This model computation proves that the integral (2.16) concentrates asymptotically on the set $\xi_{1}=0$, $x_{j+1}=x_{\ell}+v_{\ell}\left(t-t_{1}\right)$ : particles $\ell$ and $j+1$ eventually collide at the same point. The integral over $s_{1}$ also becomes an integral over the whole set $\mathbb{R}^{+}$as $\varepsilon \rightarrow 0$, and all factors $\tau_{1}=t_{1}-\varepsilon s_{1}$ tend to simply become $\tau_{1}=t_{1}$ : the two collision/recollision events tend to take place simultaneously. Note the crucial fact that the concentration in the variables $x_{j+1}$ and $\xi_{1}$, stemming from the first oscillating factor, happens independently of the variables $s_{1}, k_{1}$, and $v_{j+1}$ of the second oscillating factor. Hence we recover the asymptotics,

$$
\begin{aligned}
& \mathcal{I}_{4}^{\ell, \ell, j+1} \sim i^{3} \sum_{\sigma_{1}, \sigma_{1}^{\prime}= \pm 1} \sigma_{1} \sigma_{1}^{\prime} \int_{0}^{t} d t_{1} \int_{0}^{+\infty} d s_{1} \int d v_{j+1} \int \frac{d k_{1}}{(2 \pi)^{3}} \\
& \left|\hat{\phi}\left(k_{1}\right)\right|^{2} \exp \left(-i s_{1} k_{1} \cdot\left(v_{\ell}-v_{j+1}+\sigma_{1} k_{1}\right)\right) \\
& f_{j+1}^{0}\left(x_{1}-v_{1} t, \ldots, x_{\ell}-v_{\ell} t-\frac{\left(\sigma_{1}-\sigma_{1}^{\prime}\right) k_{1}}{2} t_{1}, \ldots, x_{j+1}-v_{j+1} t+\frac{\left(\sigma_{1}-\sigma_{1}^{\prime}\right) k_{1}}{2} t_{1} ;\right. \\
& \left.v_{1}, \ldots, v_{\ell}+\frac{\left(\sigma_{1}-\sigma_{1}^{\prime}\right) k_{1}}{2}, \ldots, v_{j+1}-\frac{\left(\sigma_{1}-\sigma_{1}^{\prime}\right) k_{1}}{2}\right) .
\end{aligned}
$$

There remains to use the fact that,

$$
\operatorname{Re} \int_{0}^{\infty} d s_{1} \exp \left(-i s_{1} k_{1} \cdot\left(v_{\ell}-v_{j+1}+\sigma_{1} k_{1}\right)\right)=-\pi \delta\left(k_{1} \cdot\left(v_{\ell}-v_{j+1}+\sigma_{1} k_{1}\right)\right) .
$$

Using formula (2.22) together with (2.21), and explicitly performing the sum over $\sigma_{1}$, it turns out that the term $|\hat{\phi}(\ldots)|^{2} \exp (\ldots)$ in (2.21) becomes merely

$$
\left|\hat{\phi}\left(k_{1}\right)\right|^{2} \delta\left(k_{1} \cdot\left(v_{\ell}-v_{j+1}+\sigma_{1} k_{1}\right)\right)
$$


as we prove in the next section. This factor gives the natural cross-section in the limiting Boltzmann equation: it gives the Fermi Golden Rule together with the natural conservation of kinetic energy.

As it is clear, the analysis performed in the present paper heavily relies on the repeated use of the stationary phase Theorem, together with asymptotic statements of the form (2.17), (2.18), and (2.19). Eventually, everything boils down to checking that various oscillating factors involve various independent variables, so as to be able to use that $\exp \left(i x^{2} / \varepsilon\right)$, respectively $\exp \left(i s x^{2}\right)$, has size $\varepsilon^{d / 2}$, respectively $s^{-d / 2}$, in the sense of distributions in $x \in \mathbb{R}^{d}$. This kind of observation is standard in the field, and we may quote [Sp1], [EY1], [EY2], [Ca1], [Ca2] for a systematic use. We also redirect the reader to the next section for precise statements in the general case.

\section{Convergence.}

The main result of this paper is summarized in the following theorem:

Theorem 3.1. Suppose $\hat{\phi} \in L^{1}\left(\mathbb{R}^{3}\right) \cap L^{\infty}\left(\mathbb{R}^{3}\right)$. Assume $f_{0}$ is such that

$$
\int\left|\hat{f}_{0}(h, k)\right| d h d k+\int d h \sup _{k \in \mathbb{R}^{3}}\left|\hat{f}_{0}(h, k)\right|<+\infty .
$$

Then for all $j \geq 1$ and for $t<t_{0}$, with $t_{0}$ sufficiently small depending on $f_{0}$ and $\phi$,

$$
\lim _{N \rightarrow \infty} \tilde{f}_{j}^{N}(t)=f^{\otimes j}(t)
$$

pointwise in $X_{j}, V_{j}, t$. The function $f(t), t \in\left[0, t_{0}\right)$ is the solution of the "classical" Boltzmann equation

$$
\begin{gathered}
\left(\partial_{t}+v \cdot \nabla_{x}\right) f=Q(f, f) \\
Q(f, f)=\iint d v_{1} d \omega B\left(\omega,\left|v-v_{1}\right|\right)\left(f^{\prime} f_{1}^{\prime}-f f_{1}\right)
\end{gathered}
$$

with $B$ given by

$$
B(\omega, w)=\frac{1}{8 \pi^{2}}|\omega \cdot w||\hat{\phi}(\omega(\omega \cdot w))|^{2} .
$$

\section{Remarks}

The bounds $\hat{f}^{0}$ in $L^{1}\left(\mathbb{R}^{d} ; L^{1} \cap L^{\infty}\left(\mathbb{R}^{d}\right)\right)$ in the statement of the Theorem directly stems from estimates of the type (2.19) above.

The assumption $\hat{\phi} \in L^{1}\left(\mathbb{R}^{3}\right) \cap L^{\infty}\left(\mathbb{R}^{3}\right)$ is implied by the stronger $\phi \in C_{0}^{k}\left(\mathbb{R}^{3}\right)$, whenever $k>3$.

Finally, the above theorem holds in any space dimension $d \geq 2$, as it is clear from the proof.

Proof: For sake of shortness we write explicitly the proof for $j=1$. The extension to arbitrary $j$ is straightforward, as well as the factorization property of the limit, which follows from Lanford's classical argument (see [L] and [CIP]). 
For $j=1$ we can write $f_{1}^{\varepsilon}=\tilde{f}_{1}^{N}$ as follows with $\ell_{1}=1$

$$
\begin{aligned}
& f_{1}^{\varepsilon}\left(x_{1}, v_{1}, t\right)=\sum_{n=0}^{N-1} \frac{(N-1)(N-2) \cdots(N-n-1)}{\varepsilon^{n}} \sum_{\ell_{2}=1}^{2} \cdots \sum_{\ell_{n}=1}^{n} \int_{0}^{t} d t_{1} \int_{0}^{t_{1}} d \tau_{1} \\
& \int_{0}^{\tau_{1}} d t_{2} \int_{0}^{t_{2}} d \tau_{2} \cdots \int_{0}^{\tau_{n-1}} d t_{n} \int_{0}^{t_{n}} d \tau_{n} S\left(t-t_{1}\right) C_{\ell_{1}, 2}^{\varepsilon} S\left(t_{1}-\tau_{1}\right) T_{\ell_{1}, 2}^{\varepsilon} \\
& S\left(\tau_{1}-t_{2}\right) C_{\ell_{2}, 3}^{\varepsilon} S\left(t_{2}-\tau_{2}\right) T_{\ell_{2}, 2}^{\varepsilon} \ldots S\left(\tau_{n-1}-t_{n}\right) C_{\ell_{n}, n+1}^{\varepsilon} S\left(t_{n}-\tau_{n}\right) T_{\ell_{n}, n+1}^{\varepsilon} S\left(\tau_{n}\right) f_{n+1}^{0} .
\end{aligned}
$$

The solution to the Boltzmann equation to be compared with the expansion (3.1) is

$$
\begin{aligned}
& f_{1}\left(x_{1}, v_{1}, t\right)=\sum_{n \geq 0} \sum_{\ell_{2}=1}^{2} \cdots \sum_{\ell_{n}=1}^{n} \int_{0}^{t} d t_{1} \cdots \int_{0}^{t_{n-1}} d t_{n} S\left(t-t_{1}\right) C_{\ell_{1}, 2} \\
& S\left(t_{1}-t_{2}\right) C_{\ell_{2}, 3} \ldots S\left(t_{n-1}-t_{n}\right) C_{\ell_{n}, n+1} S\left(t_{n}\right) f_{n+1}^{0}
\end{aligned}
$$

with

$$
\begin{aligned}
& \left(C_{\ell, j+1} f_{j+1}\right)\left(x_{1}, v_{1}, \ldots, x_{j}, v_{j}\right)=\int_{\mathbb{R}^{3}} d v_{j+1} \int_{\mathbb{S}_{2}} d \omega B\left(\omega, w_{\ell}\right) \\
& {\left[f_{j+1}\left(x_{1}, \ldots, x_{\ell}, \ldots, x_{j}, x_{\ell} ; v_{1}, \ldots, v_{\ell}-\omega\left(\omega \cdot w_{\ell}\right), \ldots, v_{j}, v_{j+1}+\omega\left(\omega \cdot w_{\ell}\right)\right)\right.} \\
& \left.\left.-f_{j+1}\left(x_{1}, \ldots, x_{\ell}, \ldots, x_{j}, x_{\ell} ; v_{1}, \ldots, v_{\ell}, \ldots, v_{j}, v_{j+1}\right)\right)\right]
\end{aligned}
$$

where $\mathbb{S}_{2}$ is the unit sphere in $\mathbb{R}^{3}, w_{\ell}=v_{\ell}-v_{j+1}$, and $B(\omega, w)$ is the quantum cross section computed in the Born approximation.

Therefore both $f_{1}^{\varepsilon}$ and $f_{1}$ are expressed as sums of integrals of $f_{n+1}^{0}$ computed in suitable points which we construct in the following way: for a fixed $n$ we call graph of order $n$ any sequence $\ell_{1}, \ldots, \ell_{n}$ with $\ell_{j} \in\{1,2, \ldots, j\}$ and denote by $\sum_{\ell_{1}, \ldots, \ell_{n}}^{\prime}$ the sum on all the possible graphs. Therefore upon interverting the $t$ and $\tau$ integrals in (3.1) for later convenience,

$$
\begin{aligned}
& f_{1}^{\varepsilon}\left(x_{1}, v_{1}, t\right)=\sum_{n \geq 0} \sum_{\ell_{1}, \ldots, \ell_{n}}^{\prime} \frac{(N-1)(N-2) \cdots(N-n-1)}{\varepsilon^{n}}(-i)^{2 n} \\
& \int_{0}^{t} d t_{1} \int_{0}^{t_{1}} d t_{2} \cdots \int_{0}^{t_{n-1}} d t_{n} \int_{t_{2}}^{t_{1}} d \tau_{1} \int_{t_{3}}^{t_{2}} d \tau_{2} \cdots \int_{t_{n}}^{t_{n-1}} d \tau_{n-1} \int_{0}^{t_{n}} d \tau_{n} \\
& \quad \sum_{\sigma_{1}, \ldots, \sigma_{n}, \sigma_{1}^{\prime}, \ldots, \sigma_{n}^{\prime}= \pm 1} \prod_{j=1}^{n} \sigma_{j} \sigma_{j}^{\prime} \int \frac{d h_{1}}{(2 \pi)^{3}} \cdots \int \frac{d h_{n}}{(2 \pi)^{3}} \int \frac{d k_{1}}{(2 \pi)^{3}} \cdots \int \frac{d k_{n}}{(2 \pi)^{3}} \prod_{j=1}^{n} \hat{\phi}\left(h_{j}\right) \hat{\phi}\left(k_{j}\right) \\
& \int_{j} d x_{2} \cdots \int d x_{n+1} \int d v_{2} \cdots \int d v_{n+1} f_{n+1}^{0}\left(y_{1}, \ldots, y_{n+1} ; u_{1}, \ldots, u_{n+1}\right) \\
& \prod_{j=1}^{n} \exp \left[i \frac{h_{j}}{\varepsilon} \cdot\left(y_{\ell_{j}}\left(t_{j}\right)-y_{j+1}\left(t_{j}\right)\right)+i \frac{k_{j}}{\varepsilon} \cdot\left(y_{\ell_{j}}\left(\tau_{j}\right)-y_{j+1}\left(\tau_{j}\right)\right)\right] .
\end{aligned}
$$

In (3.4) $y_{j}(s), u_{j}(s)$ are the backward trajectory of the particle $j \geq 1$ and its velocity. The particle $j$ is born at time $t_{j-1}$ at $x_{j}$ with velocity $v_{j}$ and the trajectory is computed for 
$s \in\left[0, t_{j-1}\right]$, (as the reader may easily check), by the following formulae: we set $t_{0}=\tau_{0}=t$, $t_{n+1}=0$ and

$$
\begin{array}{ll}
u_{j+1}\left(t_{j}\right)=v_{j+1}, & \\
u_{j+1}\left(\tau_{j}\right)=u_{j+1}\left(t_{j}\right)+\sigma_{j} \frac{h_{j}}{2} & u_{\ell_{j}}\left(\tau_{j}\right)=u_{\ell_{j}}\left(t_{j}\right)-\sigma_{j} \frac{h_{j}}{2}, \\
u_{j+1}\left(t_{j+1}\right)=u_{j+1}\left(\tau_{j}\right)+\sigma_{j}^{\prime} \frac{k_{j}}{2} & u_{l_{j}}\left(t_{j+1}\right)=u_{\ell_{j}}\left(\tau_{j}\right)-\sigma_{j}^{\prime} \frac{k_{j}}{2}, \\
u_{r}\left(t_{j+1}\right)=u_{r}\left(\tau_{j}\right)=u_{r}\left(t_{j}\right) \text { if } r \neq \ell_{j} . &
\end{array}
$$

Also, the values of the $u_{j}$ 's are extended outside the "collision" times given by the $t_{j}$ 's and $\tau_{j}$ 's, as follows,

$$
u_{j}(s)= \begin{cases}u_{j}\left(\tau_{r}\right) & \text { if } \tau_{r} \leq s<t_{r}, \\ u_{j}\left(t_{r+1}\right) & \text { if } t_{r+1} \leq s<\tau_{r} .\end{cases}
$$

The trajectories $y_{j}$ themselves are now simply defined by,

$$
y_{j}(s)=x_{j}-\int_{s}^{t_{j-1}} u_{j}(\tau) d \tau .
$$

With this construction $u_{j}(s)$ is right-continuous, i.e. at the times $t_{r}$ and $\tau_{r}$, the velocities are the outgoing ones. We have also set

$$
y_{j}=y_{j}(0), \quad u_{j}=u_{j}(0) .
$$

We denote by $\mathcal{T}^{\varepsilon}\left(t_{1}, \ldots, t_{n} ; \ell_{1}, \ldots, \ell_{n}\right)$ the contribution to the $n$-th order term of the above expansion due to the graph $\left\{\ell_{1}, \ldots, \ell_{n}\right\}$ :

$$
\begin{aligned}
& \mathcal{T}^{\varepsilon}\left(t_{1}, \ldots, t_{n} ; \ell_{1}, \ldots, \ell_{n}\right)=(-i)^{2 n} \frac{(N-1)(N-2) \cdots(N-n-1)}{\varepsilon^{n}} \int_{t_{2}}^{t_{1}} d \tau_{1} \ldots \\
& \int_{t_{n}}^{t_{n-1}} d \tau_{n-1} \int_{0}^{t_{n}} d \tau_{n} \sum_{\sigma_{1}, \ldots, \sigma_{n}, \sigma_{1}^{\prime}, \ldots, \sigma_{n}^{\prime}= \pm 1} \prod_{j=1}^{n} \sigma_{j} \sigma_{j}^{\prime} \\
& \int \frac{d h_{1}}{(2 \pi)^{3}} \cdots \int \frac{d h_{n}}{(2 \pi)^{3}} \int \frac{d k_{1}}{(2 \pi)^{3}} \cdots \int \frac{d k_{n}}{(2 \pi)^{3}} \prod_{j=1}^{n} \hat{\phi}\left(h_{j}\right) \hat{\phi}\left(k_{j}\right) \\
& \int d x_{2} \cdots \int d x_{n+1} \int d v_{2} \cdots \int d v_{n+1} f_{n+1}^{0}\left(y_{1}, \ldots, y_{n+1} ; u_{1}, \ldots, u_{n+1}\right) \\
& \prod_{j=1}^{n} \exp \left[i \frac{h_{j}}{\varepsilon} \cdot\left(y_{\ell_{j}}\left(t_{j}\right)-y_{j+1}\left(t_{j}\right)\right)+i \frac{k_{j}}{\varepsilon} \cdot\left(y_{\ell_{j}}\left(\tau_{j}\right)-y_{j+1}\left(\tau_{j}\right)\right)\right],
\end{aligned}
$$

so that

$$
f_{1}^{\varepsilon}(t)=\sum_{n \geq 0} \sum_{\ell_{1}, \ldots, \ell_{n}}^{\prime} \int_{0}^{t} d t_{1} \ldots \int_{0}^{t_{n-1}} d t_{n} \mathcal{T}^{\varepsilon}\left(t_{1}, \ldots, t_{n} ; \ell_{1}, \ldots, \ell_{n}\right)
$$

Analogously, we write

$$
f_{1}(t)=\sum_{n \geq 0} \sum_{\ell_{1}, \ldots, \ell_{n}}^{\prime} \int_{0}^{t} d t_{1} \ldots \int_{0}^{t_{n-1}} d t_{n} \mathcal{T}\left(t_{1}, \ldots, t_{n} ; \ell_{1}, \ldots, \ell_{n}\right)
$$


with

$$
\begin{aligned}
& \mathcal{T}\left(t_{1}, \ldots, t_{n} ; \ell_{1}, \ldots, \ell_{n}\right)=\sum_{\sigma \in\{-1,1\}^{n}} \int d v_{2} \cdots \int d v_{n+1} \int_{\mathbb{S}_{2}} d \omega_{2} \cdots \int_{\mathbb{S}_{2}} d \omega_{n+1} \\
& \left(\prod_{j=1}^{n} \sigma_{j} B\left(\omega_{j}, w_{j}\right)\right) f_{n+1}^{0}\left(y_{1}^{c l}, \ldots, y_{n+1}^{c l} ; u_{1}^{c l}, \ldots, u_{n+1}^{c l}\right),
\end{aligned}
$$

where $w_{j}=u_{l_{j}}^{c l}\left(t_{j}\right)-u_{j+1}^{c l}\left(t_{j}\right)$, and the classical trajectories $y_{j}^{c l}(s)$ are computed (as the reader may easily check), by the following formulae: we set $t_{0}=t, t_{n+1}=0$, and

$$
\begin{aligned}
& u_{j+1}^{c l}\left(t_{j}\right)=v_{j+1}, \\
& u_{j+1}^{c l}\left(t_{j+1}\right)=u_{j+1}^{c l}\left(t_{j}\right)+\frac{1+\sigma_{j}}{2}\left(\omega_{j} \cdot\left(u_{\ell_{j}}^{c l}\left(t_{j}\right)-u_{j+1}\left(t_{j}\right)\right) \omega_{j}\right. \\
& u_{\ell_{j}}^{c l}\left(t_{j+1}\right)=u_{\ell_{j}}^{c l}\left(t_{j}\right)-\frac{1+\sigma_{j}}{2}\left(\omega_{j} \cdot\left(u_{\ell_{j}}^{c l}\left(t_{j}\right)-u_{j+1}^{c l}\left(t_{j}\right)\right) \omega_{j}\right. \\
& u_{r}^{c l}\left(t_{j+1}\right)=u_{r}^{c l}\left(t_{j}\right) \quad \text { if } r \neq \ell_{j} .
\end{aligned}
$$

Also, the values of the $u_{j}^{c l}$ 's are extended outside the "collision" times given by the $t_{j}$ 's, as follows,

$$
u_{j}^{c l}(s)=u_{j}^{c l}\left(t_{r+1}\right) \quad \text { if } t_{r+1} \leq s<t_{r} .
$$

The trajectories $y_{j}^{c l}$ themselves are now simply defined by,

$$
y_{j}^{c l}(s)=x_{j}-\int_{s}^{t_{j-1}} u_{j}^{c l}(\tau) d \tau .
$$

Note that, because of the factors $\left(1+\sigma_{r}\right) / 2$, when $\sigma_{r}=-1$ the velocity is unchanged at time $t_{r}$, so producing a loss term, while when $\sigma_{r}=1$, the velocity changes by a term such that the conservation of energy is ensured. Finally,

$$
y_{j}^{c l}=y_{j}^{c l}(0), \quad u_{j}^{c l}=u_{j}^{c l}(0) .
$$

As stated in Theorem 3.1, we want to prove that for all $\left(x_{1}, v_{1}\right)$,

$$
f_{1}^{N}(t) \rightarrow f_{1}(t)
$$

for $t$ sufficiently small, assuming that:

1) $\hat{\phi} \in L^{1}\left(\mathbb{R}^{3}\right) \cap L^{\infty}\left(\mathbb{R}^{3}\right)$,

2) The following norms are finite

$$
N_{1}\left(f^{0}\right)=\left\|\hat{f}^{0}\right\|_{L_{1}\left(\mathbb{R}^{3} \times \mathbb{R}^{3}\right)}, \quad N_{2}\left(f^{0}\right)=\int d \xi \sup _{k}\left|\hat{f}^{0}(\xi, k)\right| .
$$

This is obtained from two steps: The first step is to prove that $\mathcal{T}^{\varepsilon}$ if uniformly bounded (Proposition 3.2), the second is to prove Eq. (3.17) via the dominated convergence theorem, ensured by Proposition 3.3. 
Proposition 3.2: (Uniform bound). There is $C>0$, only depending on $\phi$ as in Theorem 3.1, such that

$$
\left|\mathcal{T}^{\varepsilon}\left(t_{1}, \ldots, t_{n} ; \ell_{1}, \ldots, \ell_{n}\right)\right| \leq C^{n}\left(N_{1}\left(f^{0}\right)+N_{2}\left(f^{0}\right)\right)^{n}
$$

Proof: The proof relies essentially on (a systematic use of) the change of variables (2.15) and the bound (2.19) used to treat the term $\mathcal{I}_{4}^{\ell, \ell, j+1}$ of the previous section.

First we make the following change of variables

$$
\xi_{j}=\frac{h_{j}+k_{j}}{\varepsilon}, \quad s_{j}=\frac{t_{j}-\tau_{j}}{\varepsilon} .
$$

The expression of $T^{\varepsilon}\left(t_{1}, \ldots, t_{n} ; \ell_{1}, \ldots, \ell_{n}\right)$ thus becomes:

$$
\begin{aligned}
& \mathcal{T}^{\varepsilon}\left(t_{1}, \ldots, t_{n} ; \ell_{1}, \ldots, \ell_{n}\right)=(-i)^{2 n}(N-1)(N-2) \cdots(N-n-1) \varepsilon^{3 n} \\
& \int_{0}^{\left(t_{1}-t_{2}\right) / \varepsilon} d s_{1} \int_{0}^{\left(t_{2}-t_{3}\right) / \varepsilon} d s_{2} \cdots \int_{0}^{\left(t_{n-1}-t_{n}\right) / \varepsilon} d s_{n-1} \int_{0}^{t_{n} / \varepsilon} d s_{n} \sum_{\sigma_{1}, \ldots, \sigma_{n}, \sigma_{1}^{\prime}, \ldots, \sigma_{n}= \pm 1} \prod_{j=1}^{n} \sigma_{j} \sigma_{j}^{\prime} \\
& \int \frac{d \xi_{1}}{(2 \pi)^{3}} \cdots \int \frac{d \xi_{n}}{(2 \pi)^{3}} \int \frac{d k_{1}}{(2 \pi)^{3}} \cdots \int \frac{d k_{n}}{(2 \pi)^{3}} \prod_{j=1}^{n} \hat{\phi}\left(k_{j}\right) \hat{\phi}\left(-k_{j}+\varepsilon \xi_{j}\right) \\
& \int d x_{2} \cdots \int d x_{n+1} \int d v_{2} \cdots \int d v_{n+1} f_{n+1}^{0}\left(y_{1}, \ldots, y_{n+1} ; u_{1}, \ldots, u_{n+1}\right) \\
& \prod_{j=1}^{n} \exp \left[i \xi_{j} \cdot\left(y_{\ell_{j}}\left(t_{j}\right)-y_{j+1}\left(t_{j}\right)\right)\right] \\
& \prod_{j=1}^{n} \exp \left[-i \frac{k_{j}}{\varepsilon} \cdot\left[\left(y_{\ell_{j}}\left(t_{j}\right)-y_{j+1}\left(t_{j}\right)\right)-\left(y_{\ell_{j}}\left(t_{j}-\varepsilon s_{j}\right)-y_{j+1}\left(t_{j}-\varepsilon s_{j}\right)\right)\right]\right] .
\end{aligned}
$$

Also, for fixed $x_{1}, v_{1}$, and fixed $\xi_{r}, h_{r}, s_{r}, t_{r}, \sigma_{r}, \sigma_{r}^{\prime}(r=1, \ldots, n)$, we define the integral

$$
\begin{aligned}
& I=\int d X d V \prod_{j=1}^{n} \hat{\phi}\left(k_{j}\right) \hat{\phi}\left(-k_{j}+\varepsilon \xi_{j}\right) f_{n+1}^{0}\left(y_{1}, \ldots, y_{n+1} ; u_{1}, \ldots, u_{n+1}\right) \\
& \prod_{j=1}^{n} \exp \left[i \xi_{j} \cdot\left(y_{\ell_{j}}\left(t_{j}\right)-y_{j+1}\left(t_{j}\right)\right)\right] \\
& \prod_{j=1}^{n} \exp \left[-i \frac{k_{j}}{\varepsilon} \cdot\left[\left(y_{\ell_{j}}\left(t_{j}\right)-y_{j+1}\left(t_{j}\right)\right)-\left(y_{\ell_{j}}\left(t_{j}-\varepsilon s_{j}\right)-y_{j+1}\left(t_{j}-\varepsilon s_{j}\right)\right)\right]\right] .
\end{aligned}
$$

Here we use the shorthand notation,

$$
X=\left(x_{2}, \ldots, x_{n+1}\right), V=\left(v_{2}, \ldots, v_{n+1}\right), \Xi=\left(\xi_{1}, \ldots, \xi_{n}\right), K=\left(k_{1}, \ldots, k_{n}\right) .
$$

In order to bound $\mathcal{T}^{\varepsilon}$ (or $I$ ), we now follow the same lines as in the treatment of $\mathcal{I}_{4}^{\ell, \ell, j+1}$ : we use appropriate changes of variables so as to come up with factors of the form (2.17). Then we treat them as in (2.18), (2.19), (2.20). The description of the relevant changes 
of variables is the reason for the discussion made in equations (3.22) to (3.33) below. The resulting Proposition 3.3 below is analogous to the bound (2.19), and Proposition 3.4 states a generalization of the asymptotics (2.18).

Let us come to the details. We note that the mapping

$$
\left(x_{2}, v_{2}, \ldots, x_{n+1}, v_{n+1}\right) \longrightarrow\left(y_{2}, u_{2}, \ldots, y_{n+1}, u_{n+1}\right)
$$

is one-to-one with unitary Jacobian. Moreover, from Eq.s (3.7),(3.8), we have,

$$
y_{j}(s)-\left(y_{j}+u_{j} s\right)=\int_{0}^{s}\left(u_{j}(\tau)-u_{j}\right) d \tau,
$$

so that one readily obtains

$$
\begin{aligned}
& y_{\ell_{j}}\left(t_{j}\right)-y_{j+1}\left(t_{j}\right)=y_{\ell_{j}}-y_{j+1}+\left(u_{\ell_{j}}-u_{j+1}\right) t_{j}+\gamma_{j}^{1}, \\
& y_{\ell_{j}}\left(t_{j}\right)-y_{j+1}\left(t_{j}\right)-\left(y_{\ell_{j}}\left(t_{j}-\varepsilon s_{j}\right)-y_{j+1}\left(t_{j}-\varepsilon s_{j}\right)\right)=\varepsilon s_{j}\left(u_{\ell_{j}}-u_{j+1}\right)+\gamma_{j}^{2} .
\end{aligned}
$$

Here $\gamma_{j}^{i}, i=1,2$ do not depend on $y_{r}, u_{r}$ for $r=2, \ldots, n+1$. The terms $\gamma_{i}^{j}$ actually are linear in the variables $\varepsilon \xi_{r}$ and $h_{r}$, for the values $r=j, j+1, \ldots, n$. This gives in (3.21),

$$
\begin{aligned}
& I=\int d Y d U\left(\prod_{j=1}^{n} \hat{\phi}\left(k_{j}\right) \hat{\phi}\left(-k_{j}+\varepsilon \xi_{j}\right)\right) \mathrm{e}^{i \Gamma(\Xi, K)} f_{1}^{0}\left(y_{1}, u_{1}\right) f_{n}^{0}(Y, U) \\
& \prod_{j=1}^{n} \exp \left[i \xi_{j} \cdot\left(y_{\ell_{j}}-y_{j+1}\right)\right] \exp \left[i\left(u_{\ell_{j}}-u_{j+1}\right) \cdot\left(-s_{j} k_{j}+t_{j} \xi_{j}\right)\right],
\end{aligned}
$$

where

$$
\Gamma(\Xi, K):=\sum_{j=1}^{n}\left(\gamma_{j}^{1} \cdot \xi_{j}-\varepsilon^{-1} \gamma_{j}^{2} \cdot k_{j}\right),
$$

and we use the shorthand notation

$$
Y=\left(y_{2}, \ldots, y_{n+1}\right), \quad U=\left(u_{2}, \ldots, u_{n+1}\right)
$$

Now, as in (2.20), we explicitly compute the $d Y d U$ integral in (3.26). It is crucial at this stage that $\left(y_{1}, v_{1}\right)$ does not depend upon $Y$ nor $U$, and that the phase $\Gamma / \varepsilon$ does not depend on $Y$ nor $U$ neither. Also, we need the following observation (putting the variables $y_{1}$ and $u_{1}$ apart):

$$
\begin{gathered}
\sum_{j=1}^{n} \xi_{j} \cdot\left(y_{\ell_{j}}-y_{j+1}\right)=(\Xi, A Y)+\left(\sum_{j=1}^{n} \delta_{\ell_{1}, j} \xi_{j}\right) \cdot y_{1}, \\
\sum_{j=1}^{n} \xi_{j} \cdot\left(u_{\ell_{j}}-u_{j+1}\right) t_{j}=(T \Xi, A U)+\left(\sum_{j=1}^{n} \delta_{\ell_{1}, j} t_{j} \xi_{j}\right) \cdot u_{1}, \\
\sum_{j=1}^{n} s_{j} k_{j} \cdot\left(u_{\ell_{j}}-u_{j+1}\right)=(S K, A U)+\left(\sum_{j=1}^{n} \delta_{\ell_{1}, j} s_{j} k_{j}\right) \cdot u_{1},
\end{gathered}
$$


where $A, T, S$ are $n \times n$ matrices, whose elements are given by:

$$
\begin{gathered}
A_{r, s}=-\delta_{r, s}+\delta_{\ell_{r}, s+1}, \\
T=\operatorname{diag}\left(t_{1}, \ldots, t_{n}\right), \quad S=\operatorname{diag}\left(s_{1}, \ldots, s_{n}\right),
\end{gathered}
$$

and $(\cdot, \cdot)$ denotes the inner product in $\mathbb{R}^{3 n}$. The matrix $A$ is upper triangular, with -1 coefficients on the diagonal. With these notations we write,

$$
\begin{aligned}
I= & \int d Y d U\left(\prod_{j=1}^{n} \hat{\phi}\left(k_{j}\right) \hat{\phi}\left(-k_{j}+\xi_{j}\right)\right) \mathrm{e}^{\tilde{i} \widetilde{\Gamma}(\Xi, K)} f_{1}^{0}\left(y_{1}, u_{1}\right) f_{n}^{0}(Y, U) \\
& \exp [i(\Xi, A Y)] \exp [-i(S K, A U)+i(T \Xi, A U)] .
\end{aligned}
$$

Here we have set,

$$
\widetilde{\Gamma}(\Xi, K)=\Gamma(\Xi, K)+\sum_{j=1}^{n} \delta_{l_{1}, j}\left[\xi_{j} \cdot\left(y_{1}+t_{j} u_{1}\right)-s_{j} k_{j} \cdot u_{1}\right]
$$

The $d Y d U$ integral is now easily computed,

$$
I=\mathrm{e}^{\tilde{i \Gamma}(\varepsilon \Xi, K)} f_{1}^{0}\left(y_{1}, u_{1}\right)\left(\prod_{j=1}^{n} \hat{\phi}\left(k_{j}\right) \hat{\phi}\left(-k_{j}+\varepsilon \xi_{j}\right)\right) \widehat{f}_{n}^{0}\left(-A^{T} \Xi, A^{T} S K-A^{T} T \Xi\right) .
$$

Armed with expression (3.34) for $I$, we are ready to give uniform bounds on this term, as well as convergence results for $\mathcal{T}^{\varepsilon}$.

Proposition 3.3. Let us define the function

$$
g(\Xi, K):=\left|\left(\prod_{j=1}^{n} \hat{\phi}\left(k_{j}\right)\right) \widehat{f}_{n}^{0}\left(-A^{T} \Xi, A^{T} S K-A^{T} T \Xi\right)\right|
$$

which is independent of $\varepsilon$ (the dependence of $g$ upon the $s_{j}$ 's and $t_{j}$ 's is not made explicit). Then, the following estimate holds true,

$$
\int_{\mathbb{R}^{6 n}} d \Xi d K g(\Xi, K) \leq C^{n}\left(N_{1}\left(f^{0}\right)+N_{2}\left(f^{0}\right)\right)^{n} \prod_{j=1}^{n} \frac{1}{\left(1+s_{j}\right)^{3}}
$$

for some constant $C>0$ depending on $\phi$. In particular, I defined by (3.34) satisfies,

$$
\left|\int_{\mathbb{R}^{6 n}} I d \Xi d K\right| \leq C^{n}\left\|f^{0}\right\|_{L^{\infty}\left(\mathbb{R}^{3}\right)}\left(N_{1}\left(f^{0}\right)+N_{2}\left(f^{0}\right)\right)^{n} \prod_{j=1}^{n} \frac{1}{\left(1+s_{j}\right)^{3}} .
$$

Hence $I$ is uniformly (in $\varepsilon$ ) integrable with respect to the variables $s_{1} \geq 0, \ldots, s_{n} \geq 0$.

\section{Remark}

If the space dimension is $d$, the factors $\left(1+s_{j}\right)^{-3}$ above become $\left(1+s_{j}\right)^{-d}$. 
Proof:

The following first bound obviously follows from (3.34), together with the fact that $|\operatorname{det} A|=1$ :

$$
\int d \Xi d K g(\Xi, K) \leq C\|\hat{\phi}\|_{L^{1}\left(\mathbb{R}^{3}\right)}^{n}\left\|\hat{f}_{n}^{0}\right\|_{L^{1}\left(\mathbb{R}^{d} ; L^{\infty}\left(\mathbb{R}^{d}\right)\right)}
$$

On the other hand, to recover decay estimates as the $s$-variables grow, one makes the change of variables $K \rightarrow S^{-1} K$ in (3.34) (valid if the $s_{i}$ 's are, say $\neq 0$ ), and we get,

$$
\begin{aligned}
& \int d \Xi d K g(\Xi, K) \leq\left(\prod_{j=1}^{n} s_{j}^{-3}\right) \int d \Xi d K\left(\prod_{j=1}^{n}\left|\hat{\phi}\left(\frac{k_{j}}{s_{j}}\right)\right|\right)\left|\widehat{f}_{n}^{0}\left(-A^{T} \Xi, A^{T} K-A^{T} T \Xi\right)\right| \\
& \leq C\left(\prod_{j=1}^{n} s_{j}^{-3}\right)\|\hat{\phi}\|_{L^{\infty}\left(\mathbb{R}^{3}\right)}^{n}\left\|\widehat{f}_{n}^{0}\right\|_{L^{1}\left(\mathbb{R}^{d} ; L^{1}\left(\mathbb{R}^{d}\right)\right)} \cdot
\end{aligned}
$$

All this proves the proposition.

Using the Proposition 3.3 we conclude the proof of the uniform bound (3.18): the factor

$$
(N-1)(N-2) \cdots(N-n-1) \varepsilon^{3 n}
$$

in (3.20) is bounded by $c^{n}$, and the number of terms in $\sum_{\sigma, \sigma^{\prime}}$ is $2^{2 n}$. The bound (3.18) implies the absolute uniform convergence of the series (3.4) for sufficiently small times. Indeed, the number of graphs $\left\{\ell_{1}, \ldots, \ell_{n}\right\}$ is $n$ !, while the time ordered integral gives a factor $t^{n} / n$ !. Therefore we have

$$
\left|f_{1}\left(t ; x_{1}, v_{1}\right)\right| \leq \sum_{n=0}^{\infty}\left[C\left(N_{1}\left(f^{0}\right)+N_{2}\left(f^{0}\right)\right) t\right]^{n}
$$

converging for $C\left(N_{1}\left(f^{0}\right)+N_{2}\left(f^{0}\right)\right) t<1$.

Proposition 3.4 (Term by term convergence). For all $\left(x_{1}, v_{1}\right)$, and any choice of $t>t_{1}>\cdots>t_{n}>0$ and $\left\{\ell_{1}, \ldots, \ell_{n}\right\}$ with $\ell_{j} \in\{1,2, \ldots, j\}$,

$$
\lim _{\varepsilon \rightarrow 0} \mathcal{T}^{\varepsilon}\left(t_{1}, \ldots, t_{n} ; \ell_{1}, \ldots, \ell_{n}\right)=\mathcal{T}\left(t_{1}, \ldots, t_{n} ; \ell_{1}, \ldots, \ell_{n}\right)
$$

Proof: We want to pass to the limit $\varepsilon \rightarrow 0$ in formula (3.20) giving the value of $\mathcal{T}^{\varepsilon}$. Now, equation (3.20) expresses $\mathcal{T}^{\varepsilon}$ as the integral over $s_{1}, \ldots, s_{n}$, together with $\Xi$ and $K$, of the quantity $I$ used above. Hence Proposition 3.3 makes it possible to use the dominated convergence theorem, and we can safely interchange the $\lim _{\varepsilon \rightarrow 0}$ with the integration on $\left(s_{1}, \ldots, s_{n}\right)$, and $\Xi, K$ in $(3.20)$.

Coming back to formula (3.21) expressing $I$ as an integral over variables $X$ and $V$, we may use that the function $f_{n+1}^{0}(Y, U)$ (seen as a function of $X$ and $V$ ) is a fixed (independent 
of $\varepsilon$ ) function in $L^{1}\left(\mathbb{R}^{6 n}\right)$. Hence we can again use the dominated convergence Theorem to interchange $\lim _{\varepsilon \rightarrow 0}$ with the integration on $X$ and $V$ in (3.21).

From Eq.s (3.5)-(3.7) and using $h_{j}=-k_{j}+\varepsilon \xi_{j}$, we have that $y_{j}(s) \rightarrow \bar{y}_{j}(s)$, and, for $s \neq t_{r}, u_{j}(s) \rightarrow \bar{u}_{j}(s)$, where $\bar{u}_{j}(s)$ are defined in a similar way of $u_{j}^{c l}(s)$, substituting the recursive relation $(3.13)$ with

$$
\begin{aligned}
& \bar{u}_{j+1}\left(t_{j+1}\right)=\bar{u}_{j+1}\left(t_{j}\right)+\frac{\sigma_{j}^{\prime}-\sigma_{j}}{2} k_{j} \\
& \bar{u}_{\ell_{j}}\left(t_{j+1}\right)=\bar{u}_{\ell_{j}}\left(t_{j}\right)-\frac{\sigma_{j}^{\prime}-\sigma_{j}}{2} k_{j} .
\end{aligned}
$$

The trajectories are given by

$$
\bar{y}_{j}(s)=x_{j}-\int_{s}^{t_{j-1}} \bar{u}_{j}(\tau) d \tau .
$$

Moreover, from (3.5) and the position $h_{j}=-k_{j}+\varepsilon \xi_{j}$

$$
\begin{aligned}
& \frac{y_{\ell_{j}}\left(t_{j}\right)-y_{\ell_{j}}\left(t_{j}-\varepsilon s_{j}\right)}{\varepsilon}=s_{j} u_{\ell_{j}}\left(t_{j}-\varepsilon s_{j}\right) \rightarrow s_{j}\left(\bar{u}_{\ell_{j}}\left(t_{j}\right)+\frac{\sigma_{j}}{2} k_{j}\right) \\
& \frac{y_{j+1}\left(t_{j}\right)-y_{j+1}\left(t_{j}-\varepsilon s_{j}\right)}{\varepsilon}=s_{j} u_{j+1}\left(t_{j}-\varepsilon s_{j}\right) \rightarrow s_{j}\left(\bar{u}_{j+1}\left(t_{j}\right)-\frac{\sigma_{j}}{2}\right) k_{j} .
\end{aligned}
$$

Thus, from (3.20), we obtain

$$
\begin{aligned}
& \lim _{\varepsilon \rightarrow 0} \mathcal{T}^{\varepsilon}\left(t_{1}, \ldots, t_{n} ; \ell_{1}, \ldots, \ell_{n}\right)= \\
& (-i)^{2 n} \sum_{\sigma_{1}, \ldots, \sigma_{n}, \sigma_{1}^{\prime}, \ldots, \sigma_{n}^{\prime}= \pm 1} \prod_{j=1}^{n} \sigma_{j} \sigma_{j}^{\prime} \int d V \int \frac{d K}{(2 \pi)^{3 n}} \int_{0}^{+\infty} d s_{1} \ldots \int_{0}^{+\infty} d s_{n} \\
& \int d X \int \frac{d \Xi}{(2 \pi)^{3 n}} \prod_{j=1}^{n}\left|\hat{\phi}\left(k_{j}\right)\right|^{2} f_{n+1}\left(\bar{y}_{1}, \ldots, \bar{u}_{n+1} ; \bar{u}_{1}, \ldots, \bar{y}_{n+1}\right) \\
& \prod_{j=1}^{n} \exp i\left[\xi_{j} \cdot\left(\bar{y}_{\ell_{j}}\left(t_{j}\right)-x_{j+1}\right)-k_{j} \cdot\left[\left(\bar{w}_{j}+k_{j} \sigma_{j}\right) \tau_{j}^{\prime}\right]\right],
\end{aligned}
$$

where $\bar{w}_{j}=\bar{u}_{\ell_{j}}\left(t_{j}\right)-\bar{u}_{j+1}\left(t_{j}\right)$. As indicated at the beginning of the proof, we have now come up with factors of the form (2.17). Hence, as in the previous section, there only remains to take care of the $d \Xi$ and $d s_{1} \ldots d s_{n}$ integration as we did in (2.18).

The integration on $\Xi$ produces a delta function concentrating on the set $\left\{X \mid x_{j+1}=\right.$ $\left.\bar{y}_{\ell_{j}}\left(t_{j}\right), j=1, \ldots, n\right\}$, meaning that the particle $j+1$ is born at time $t_{j}$ exactly where the ancestor is at time $t_{j}$. Let $\left\{\tilde{y}_{j}(t), j=1, \ldots, n+1\right\}$ be the trajectories $\bar{y}_{j}(t)$ satisfying this additional condition. Since such trajectories do not depend on the $s$ 's, we conclude that

$$
\begin{aligned}
& \lim _{\varepsilon \rightarrow 0} \mathcal{T}^{\varepsilon}\left(t_{1}, \ldots, t_{n} ; \ell_{1}, \ldots, \ell_{n}\right)= \\
& (-i)^{2 n} \sum_{\sigma_{1}, \ldots, \sigma_{n}, \sigma_{1}^{\prime}, \ldots, \sigma_{n}^{\prime}= \pm 1} \prod_{j=1}^{n} \sigma_{j} \sigma_{j}^{\prime} \int \frac{d K}{(2 \pi)^{3 n}} \int d V \prod_{j=1}^{n}\left|\hat{\phi}\left(k_{j}\right)\right|^{2} \\
& f_{n+1}\left(\bar{y}_{1}, \ldots, \bar{y}_{n+1} ; \bar{u}_{1}, \ldots, \bar{u}_{n+1}\right) \prod_{j=1}^{n} \int_{0}^{+\infty} d s \exp \left[-i s k_{j} \cdot\left[\left(\bar{w}_{j}+k_{j} \sigma_{j}\right)\right]\right]
\end{aligned}
$$


where the integrals on the s's make sense as distributions (see (2.20) and the bound (2.19)). There remains to explicitly compute the integral over $s$ and the sum over the $\sigma$ 's, so as to identify $\mathcal{T}\left(t_{1}, \ldots, t_{n} ; \ell_{1}, \ldots, \ell_{n}\right)$ in (3.41). Using formula (2.22) together with the fact that the expression for the limit is invariant under the transformation

$$
s \rightarrow-s, \quad k_{j} \rightarrow-k_{j}, \quad \sigma_{j} \rightarrow-\sigma_{j}, \quad \sigma_{j}^{\prime} \rightarrow-\sigma_{j}^{\prime},
$$

we can substitute

$$
\int_{0}^{+\infty} d s \exp \left[-i s k_{j} \cdot\left[\left(\bar{w}_{j}+k_{j} \sigma_{j}\right)\right]\right] \quad \text { with its real part, i.e. } \quad \pi \delta\left(k_{j} \cdot\left(\bar{w}_{j}+k_{j} \sigma_{j}\right)\right) .
$$

Doing the change of variables $\eta_{j}=-\sigma_{j} k_{j}$, and $\sigma_{j}^{\prime}=-\sigma_{j} \bar{\sigma}_{j}$, the recursive relations (3.38) become

$$
\begin{aligned}
& \bar{u}_{j+1}\left(t_{j+1}\right)=\bar{u}_{j+1}\left(t_{j}\right)+\frac{1+\bar{\sigma}_{j}}{2} \eta_{j}, \\
& \bar{u}_{\ell_{j}}\left(t_{j+1}\right)=\bar{u}_{\ell_{j}}\left(t_{j}\right)-\frac{1+\bar{\sigma}_{j}}{2} \eta_{j} .
\end{aligned}
$$

Then the sum on $\sigma_{j}$ is free and Eq. (3.41) becomes

$$
\begin{gathered}
\lim _{\varepsilon \rightarrow 0} \mathcal{T}^{\varepsilon}\left(t_{1}, \ldots, t_{n} ; \ell_{1}, \ldots, \ell_{n}\right)=\sum_{\bar{\sigma}_{1}, \ldots, \bar{\sigma}_{n}= \pm 1} \prod_{j=1}^{n} \bar{\sigma}_{j} \int \frac{d \eta_{1} \cdots d \eta_{n}}{(2 \pi)^{2 n}} \int d V \prod_{j=1}^{n}\left|\hat{\phi}\left(\eta_{j}\right)\right|^{2} \\
f_{n+1}\left(\tilde{y}_{1}, \ldots, y_{n+1} ; \bar{u}_{1}, \ldots, \bar{u}_{n+1}\right) \delta\left(\eta_{j} \cdot\left(\bar{w}_{j}-\eta_{j}\right)\right) .
\end{gathered}
$$

We perform partially the integration in $\eta_{j}$ in the following way: let $\gamma$ be a smooth function, we have, going to polar coordinates,

$$
\begin{aligned}
& \int d \eta \gamma(\eta) \delta(\eta \cdot(w-\eta))=\int_{\mathbb{S}_{2}} d \omega \int_{0}^{+\infty} d \lambda \lambda^{2} \gamma(\lambda \omega) \delta\left(\lambda \omega \cdot w-\lambda^{2}\right) \\
& \quad=\int_{\mathbb{S}_{2}} d \omega(\omega \cdot w) \gamma((\omega \cdot w) \omega) \mathbf{1}\{\omega \cdot w>0\} \\
& =\frac{1}{2} \int_{\mathbb{S}_{2}} d \omega|\omega \cdot w| \gamma((\omega \cdot w) \omega) .
\end{aligned}
$$

Inserting this identity in (3.42), we recover the classical trajectories defined in Eq.s (3.13)(3.15). Doing the integrations in $\lambda_{j}=\left|\eta_{j}\right|$, we identify the limit in Eq. (3.43) with Eq. (3.12), where

$$
B(\omega, w)=\frac{1}{8 \pi^{2}}|\omega \cdot w||\hat{\phi}((\omega \cdot w) \omega)|^{2}
$$

We remark that the cross-section we have found in the limiting Boltzmann equation is that given by the Born approximation for the Quantum Scattering problem. This is known as Fermi's Golden Rule (see [AM], [RV], [Bo], [CTDL], [Co] ...). For the low-density limit the situation is different. Now all terms in the perturbative expansion of $S_{i n t}(t)$ play the same 
role: We thus expect that the same result holds as Theorem 3.1, but with a cross section given by the full scattering matrix $\mathcal{S}$ associated to the potential $\phi$. We mention in passing the following well-known fact [RS]: $\mathcal{S}$ admits a power series expansion in the potential $\phi$, called the Born series expansion, whose first term is precisely given by the Fermi Golden Rule,

$$
\mathcal{S}(k)=|\hat{\phi}(k)|^{2}+O\left(\hat{\phi}^{3}\right)
$$

roughly. The weak coupling regime may thus be seen (technically) as some first order approximation of the low density regime. We refer to [Ni1], [Ni2] for a kinetic interpretation of the scattering matrix in a semi-classical regime. We also refer to [Ca1], [Ca2], for the derivation of a linear Boltzmann equation involving the Born series expansion in a low density regime. We finally quote $[\mathrm{Co}]$ for a physical discussion of related questions.

Some comments are in order.

We underline once more that while in the low-density regime classical and quantum systems evolve similarly according to the Boltzmann equation, the situation changes drastically in the weak-coupling limit. Here contrary to the behavior outlined in the present paper for quantum systems, classical systems of particles are expected to satisfy the Landau equation which a diffusive character (see [Sp2]). Unfortunately no rigorous result is known in this direction. The situation is better understood for linear problems. Obviously the same scalings can be also considered for a test particle moving in a random distribution of obstacles. In this context it is possible to derive a linear transport equation for a classical particle in the low-density regime (see $[\mathrm{G}],[\mathrm{BBS}]$, the review paper [Sp3], or the textbook [Sp2]) or the linear Landau equation in the weak coupling limit (see [DGL]). A linear Boltzmann equation can be derived in the weak-coupling limit either for short times (see Refs [Sp1],[La], [HLW] ) or globally in time (see [EY1], [EY2]). The one-dimensional case is somehow pathological (see $[\mathrm{EPT}]$ ). For the case of non random scatterers negative results are available [CP1], [CP2] (see also [BGW] in the classical context).

Similar considerations in the case of an atom coupled to a gas have been developed in [Dü]. In addition, notice that the problem of the wave motion in a random medium is of interest for the applications as shown in Ref.s [KPR] We finally mention $[\mathrm{PV}]$ for the analysis of a weak coupling regime when the obstacles are temporally random (and the underlying process is at once (almost) Markovian).

At the physical level, the question of passing from the Schrödinger equations to (linear or non-linear) Boltzmann equations is an old problem. We may quote [Pa], [KL1], [KL2], $[\mathrm{Ku}]$, and [VH1], [VH2], [Zw], as well as the textbooks quoted before.

When this paper was finished we were informed by H.T. Yau that arguments similar to those of the present paper have been independently developed by him and his group in a paper in preparation.

\section{REFERENCES}

[AM] N.W. Ashcroft, N.D. Mermin, Solid stats physics, Saunders, Philadelphia (1976). [Bo] A. Bohm, Quantum Mechanics, Texts and monographs in Physics, Springer-Verlag (1979).

[BBS] C. Boldrighini, L.A. Bunimovich, Ya. Sinai, On the Boltzmann equation for the Lorentz gas, J. Stat. Phys., Vol. 32, N. 3, pp. 477-501 (1983). 
[BGW] J. Bourgain, F. Golse, B. Wennberg, On the distribution of free path lengths for the periodic Lorentz gas, Comm. Math. Phys., Vol. 190, pp. 491-508 (1998).

[Ca1] F. Castella, From the von Neumann equation to the Quantum Boltzmann equation in a deterministic framework, J. Stat. Phys., Vol. 104, N. 1/2, pp. 387-447 (2001).

[Ca2] F. Castella, ¿From the von Neumann equation to the Quantum Boltzmann equation II: identifying the Born series, J. Stat. Phys., Vol. 106, N. 5/6, pp. 1197-1220 (2002).

[CP1] F. Castella, A. Plagne, A distribution result for slices of sums of squares, Math. Proc. Cambridge Philos. Soc., Vol. 132, N.1, pp. 1-22 (2002).

[CP2] F. Castella, A. Plagne, Non-derivation of the Quantum Boltzmann equation from the periodic Schrödinger equation, To appear in Indiana Univ. Math. J. (2003).

[CC] S. Chapman and T. G. Cowling, The Mathematical Theory of Non-uniform Gases, Cambridge Univ. Press, Cambridge, England (1970).

[CIP] C. Cercignani, R. Illner, M. Pulvirenti, The mathematical theory of dilute gases, Applied Mathematical Sciences, Vol. 106, Springer-Verlag, New York (1994).

[Ch] S.L. Chuang, Physics of optoelectronic devices, Wiley series in pure and applied optics, New-York (1995).

[CTDL] C. Cohen-Tannoudji, B. Diu, F. Laloë, Mécanique Quantique, I et II, Enseignement des Sciences, Vol. 16, Hermann (1973).

[Co] M. Combescot, On the generalized golden rule for transition probabilities, Phys. A: Math. Gene., Vol. 34, N. 31, pp. 6087-6104 (2001).

[Dü] R. Dümcke, The low density limit for an N-level system interacting with a free Bose or Fermi gas, Comm. Math. Phys., Vol. 97, N. 3, pp. 331-359 (1985).

[DGL] D. Dürr, S. Goldstein, J.L. Lebowitz Asymptotic motion of a classical particle in a random potential in two dimension: the Landau model, Comm. Math. Phys., N. 113, pp. 209-230 (1987).

[EY1] L. Erdös, H.T. Yau, Linear Boltzmann Equation as Scaling Limit of Quantum Lorentz Gas Advances in differential equations and mathematical physics (Atlanta, GA, 1997), pp. 137-155, Contemp. Math., 217, Amer. Math. Soc., Providence, RI (1998).

[EY2] L. Erdös, H.T. Yau, Linear Boltzmann equation as the weak coupling limit of a random Schrödinger equation, Comm. Pure Appl. Math., Vol. 53, N. 6, pp. 667-735 (2000).

[EPT] R. Esposito, M. Pulvirenti, A. Teta, The Boltzmann equation for a one-dimensional quantum Lorentz gas, Comm. Math. Phys., Vol. 204, no. 3, pp. 619-649 (1999), and Erratum: "The Boltzmann equation for a one-dimensional quantum Lorentz gas", Comm. Math. Phys., Vol. 214, N. 2, pp. 493-494 (2000).

[Fi] M.V. Fischetti, Theory of electron transport in small semiconductor devices using the Pauli master equations, J. Appl. Phys., Vol. 83, N. 1, pp. 270-291 (1998).

[G] G. Gallavotti, Time evolution problems in classical statistical mechanics and the windtree-model Cargese Lectures in Physics, vol IV, ed. D. Kastler, Gordon Breach, Paris, (1970);Divergences and approach to equilibrium in the Lorenz and the wind-tree models Phys.Rev. 185, 308-322 (1969). See also the book Statistical Mechanics, Appendix A2 to Ch. 1, Springer-Verlag (1999).

[HLW] T.G. Ho, L.J. Landau, A.J. Wilkins, On the weak coupling limit for a Fermi gas in a random potential, Rev. Math. Phys., Vol. 5, N. 2, pp. 209-298 (1993).

[Hö] L. Hörmander, The analysis of linear partial differential operators, SpringerVerlag, Berlin (1994).

[Hu] K. Huang, Statistical mechanics, Wiley and Sons (1963). 
[IP] R. Illner, M. Pulvirenti, Global Validity of the Boltzmann equation for a two dimensional rare gas in vacuum, Comm. Math. Phys., Vol. 105, pp. 189-203 (1986). Erratum and improved result Comm. Math. Phys., Vol. 121, pp. 143-146 (1989).

[KPR] J.B. Keller, G. Papanicolaou, L. Ryzhik, Transport equations for elastic and other waves in random media, Wave Motion, Vol. 24, N. 4, p. 327-370 (1996).

[KL1] W. Kohn, J.M. Luttinger, Phys. Rev., Vol. 108, pp. 590 (1957).

[KL2] W. Kohn, J.M. Luttinger, Phys. Rev., Vol. 109, pp. 1892 (1958).

[Ku] R. Kubo, J. Phys. Soc. Jap., Vol. 12 (1958).

[L] O. Lanford III, Time evolution of large classical systems, Lecture Notes in Physics, Vol. 38, pp. 1-111, E.J. Moser ed., Springer-Verlag (1975).

[La] L.J. Landau, Observation of Quantum Particles on a Large Space-Time Scale, J. Stat. Phys., Vol. 77, N. 1-2, pp. 259-309 (1994).

[MRS] P.A. Markowich, C. Ringhofer, C. Schmeiser, Semiconductor equations, Springer-Verlag, Vienna (1990).

[Ni1] F. Nier, Asymptotic Analysis of a scaled Wigner equation and Quantum Scattering, Transp. Theor. Stat. Phys., Vol. 24, N. 4 et 5, pp. 591-629 (1995).

[Ni2] F. Nier, A semi-classical picture of quantum scattering, Ann. Sci. Ec. Norm. Sup., 4. Sér., t. 29, p. 149-183 (1996).

[PV] F. Poupaud, A. Vasseur, Classical and quantum transport in random media, Preprint University of Nice (2001).

[Pa] W. Pauli, Festschrift zum 60. Geburtstage A. Sommerfelds, p. 30, Hirzel, Leipzig (1928).

[RS] M. Reed, B. Simon, Methods of modern mathematical physics III. Scattering theory, Academic Press, New York-London ( 1979).

[RV] E. Rosencher, B. Vinter, Optoelectronique, Dunod (2002).

[Sp1] H. Spohn, Derivation of the transport equation for electrons moving through random impurities, J. Stat. Phys., Vol. 17, N. 6, pp. 385-412 (1977).

[Sp2] H. Spohn, Large scale dynamics of interacting particles, Springer (1991).

[Sp3] H. Spohn, Kinetic equations from Hamiltonian dynamics: Markovian limits, Rev. Modern Phys., Vol. 52, N. 3, pp. 569-615 (1980).

[VH1] L. Van Hove, Physica, Vol. 21 p. 517 (1955).

[VH2] L. Van Hove, Physica, Vol. 23 p. 441 (1957).

[VH3] L. Van Hove, in Fundamental Problems in Statistical Mechanics, E.G.D. Cohen ed., p. 157 (1962).

$[\mathrm{Zw}]$ R. Zwanzig, Quantum Statistical Mechanics, P.H.E. Meijer ed., Gordon and Breach, New-York (1966). 